\title{
Electric-line-source illumination of a circular cylinder of lossless double-negative material: an investigation of near field, directivity, and radiation resistance
}

Arslanagic, Samel; Breinbjerg, Olav

Published in:

I E E E Antennas and Propagation Magazine

Link to article, DOI:

10.1109/MAP.2006.1703397

Publication date:

2006

Document Version

Publisher's PDF, also known as Version of record

Link back to DTU Orbit

Citation (APA):

Arslanagic, S., \& Breinbjerg, O. (2006). Electric-line-source illumination of a circular cylinder of lossless doublenegative material: an investigation of near field, directivity, and radiation resistance. I E E E Antennas and Propagation Magazine, 48(3), 38-54. https://doi.org/10.1109/MAP.2006.1703397

\section{General rights}

Copyright and moral rights for the publications made accessible in the public portal are retained by the authors and/or other copyright owners and it is a condition of accessing publications that users recognise and abide by the legal requirements associated with these rights.

- Users may download and print one copy of any publication from the public portal for the purpose of private study or research.

- You may not further distribute the material or use it for any profit-making activity or commercial gain

- You may freely distribute the URL identifying the publication in the public portal 


\title{
Electric-Line-Source lllumination of a Circular Cylinder of Lossless Double-Negative Material: An Investigation of Near Field, Directivity, and Radiation Resistance
}

\author{
Samel Arslanagić and Olav Breinbjerg \\ Ørsted DTU, Electromagnetic Systems, Technical University of Denmark \\ Building 348, Ørsteds Plads, DK-2800 Kgs. Lyngby, Denmark \\ Tel: +45 4588 1444; Fax: +45 4593 1634; E-mail: sar@oersted.dtu.dk, ob@oersted.dtu.dk
}

\begin{abstract}
This work investigates the properties of an antenna-like configuration with an electric line source radiating in the presence of a double-negative circular cylinder. First, the analytical eigenfunction-series solution is derived. Second, this solution is employed in numerical calculations to study the properties of the near field, inside as well as outside the cylinder, and the far-field. Third, the variations of these fields are examined, as well as the radiation resistance and radiation pattern, as functions of the geometrical and electromagnetic parameters of the configuration. It is demonstrated that the scattering properties of the double-negative cylinder are vastly different from those of the corresponding double-positive cylinder. In particular, the focusing effect inside the cylinder and the angular variation of the directivity exhibit distinct characteristics.
\end{abstract}

Keywords: Antennas; metamaterials; double-negative materials; permittivity; permeability; cylinders; cylindrical antennas; electromagnetic scattering

\section{Introduction}

Q uite recently, there has emerged a strong interest in exploring and exploiting the electromagnetic characteristics and properties of artificial materials, broadly referred to as metamaterials. A very interesting example of these materials is the so-called double-negative (DNG) material [1-3], in which the real parts of both the permittivity and the permeability are negative. The idea of DNG materials dates back to the late $1960 \mathrm{~s}$, when the Russian physicist, V. G. Veselago, theoretically investigated the propagation of plane electromagnetic waves in lossless, isotropic, DNG materials [1]. In such materials, the electric and magnetic field intensity vectors of a plane wave form a left-handed set of vectors with its wavenumber vector. This is in contrast to double-positive (DPS) materials, characterized by positive real parts of the permittivity and the permeability, in which these vectors form a right-handed set. In addition, the power-flow density of a plane wave in DNG materials always forms a right-handed set of vectors with the electric and magnetic field intensity vectors, obviously implying that the phase and group velocities are oppositely directed. The reversal of the phase-velocity direction relative to the direction of the power flow leads to some interesting physical phenomena, such as the reversal of the Doppler effect, the reversal of the Vavilov-Cerenkov effect, and, perhaps most importantly, the negative refraction at a plane interface separating two DPS and DNG materials [1].

It is known that DNG materials are not found in nature and must therefore be made artificially. Recently, the feasibility of constructing negative-permittivity materials, on the one hand [4], and negative permeability materials, on the other [5], was demonstrated. These two separate approaches were combined, thereby providing the first realizations of DNG materials $[2,3]$, and the first experimental verifications followed briefly thereafter $[6,7]$. These first realizations of DNG materials were based on periodic arrangements of conducting wires and split-ring resonators (SRRs). However, other realizations exist and employ, e.g., a parallel-plate waveguide filled by a two-dimensional (2D) array of broadside coupled split-ring resonators [8], transmission-line networks loaded with capacitors and inductors $[9,10]$, and magneto-dielectric spherical particles embedded in a background matrix [11]

The strong interest in DNG materials is due to their unfamiliar electromagnetic properties, and the potential applications due to these properties [1]. In particular, the lossless DNG slab has been of interest, due to its so-called "perfect lens" property [12], but cylindrical [13-16] as well as spherical configurations [16-19] 

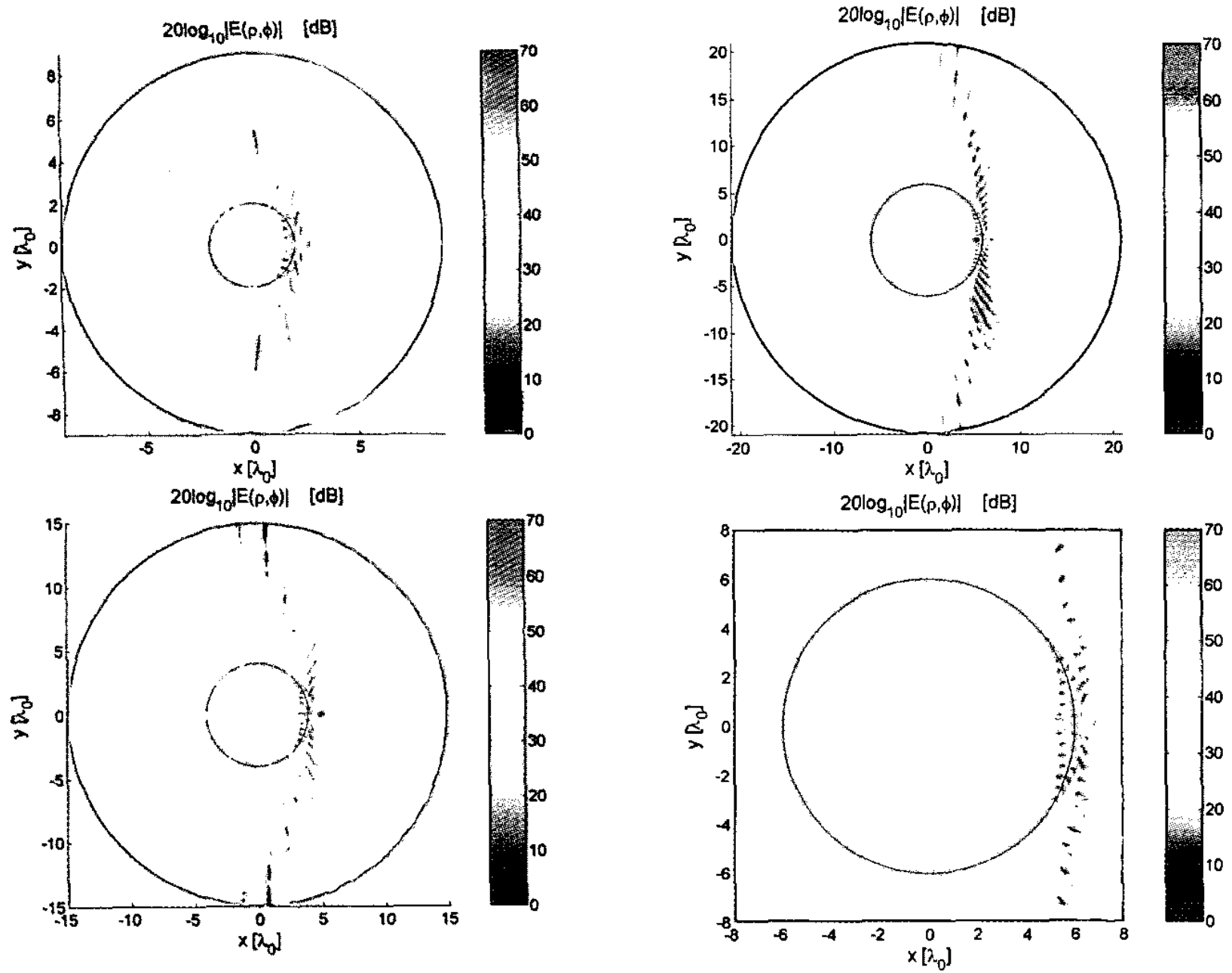

Figure 2. The electric-field distribution for " $1 \varepsilon_{0} 1 \mu_{0}$ " DNG cylinders having radii of $a=2 \lambda_{0}$ (top) and $a=4 \lambda_{0}$ (bottom). The electric line source was at a distance $b=1 \lambda_{0}$ from the cylinders.

Figure 3. (top) The electric-field distribution for a " $1 \varepsilon_{0} 1 \mu_{0}$ " DNG cylinder having a radius of $a=6 \lambda_{0}$ with the electric line source at a distance $b=1 \lambda_{0}$ from the cylinder. (bottom) $A$ selected region of the top plot, showing a closer look of the high-field-value formation. 
involving DNG materials have been investigated, as well. A considerable amount of work with regard to the development and applications of DNG materials, and metamaterials in general, has already been accomplished, as is evidenced by $[20,21]$. Nevertheless, there is a continuing interest in further exploring the properties and potential applications of these materials.

The purpose of the present work is to investigate the properties of a general antenna configuration with a DNG material. To this end, an infinitely long circular cylinder of lossless DNG material, henceforth referred to as simply a DNG cylinder, illuminated by a close-by electric line source (ELS) is chosen. (This type of illumination corresponds to TM polarization. The results for TE polarization, i.e., the magnetic-line-source illumination, are easily obtained from the duality theorem, and are thus not included here.) Although this two-dimensional configuration does not constitute a practical antenna, it possesses a finite cross section and a localized source, and may thus reveal effects that are absent in configurations with one-dimensional (1D) slabs and/or plane-wave illumination. Previous investigations of DNG cylinder configurations concerned evaluation of their scattering width under plane-wave illumination [13-16], and their cavity and waveguide properties [14].

The present manuscript is organized as follows: In Section 2, the exact analytical solutions for DPS and DNG cylinders are presented. The DNG cylinder solution is used in Section 3 in numerical calculations to investigate the influence of different geometrical and electromagnetic parameters - such as cylinder radius, electronic line source position, cylinder permittivity and permeability $\rightarrow$ on the near field, inside as well as outside the cylinder, and on the far field, in terms of the directivity and the radiation resistance. Throughout the investigations, the case of a DPS cylinder is used as a reference. The time factor $\exp (j \omega t)$, with $\omega$ being the angular frequency and $t$ being the time, is assumed and suppressed throughout the manuscript.

\section{Exact Analytical Solutions for DPS and DNG Cylinders}

The analytical results for DPS and DNG cylinders are presented in this section. The presentation comprises several steps. First, the antenna configuration is described. Second, the case of DPS cylinders is analyzed by employing the well-known eigenfunction-expansion method, and the expressions for both the scattered and transmitted fields are derived. Third, the considerations necessary to treat the corresponding DNG cylinders are outlined. In this respect, the sign of various parameters in DNG materials is briefly discussed.

\subsection{Configuration}

The configuration of interest is depicted in Figure 1. It consists of a cylinder made of a lossless simple DPS or DNG material, illuminated by an electric line source of a constant electric current, $I_{e}$ [A], placed close and parallel to the cylinder. The ambient medium is free space, i.e., its material parameters are $\left(\varepsilon_{0}, \mu_{0}\right)$, where $\varepsilon_{0}=8.854 \times 10^{-12}[\mathrm{~F} / \mathrm{m}]$ is the frec-space permittivity, and $\mu_{0}=4 \pi \times 10^{-7}[\mathrm{H} / \mathrm{m}]$ is the free-space permeability. The free-space wavenumber is $k_{0}=\omega \sqrt{\varepsilon_{0} \mu_{0}}=2 \pi / \lambda_{0}$, with $\lambda_{0}$ being the free-space wavelength, and the free-space intrinsic impedance is

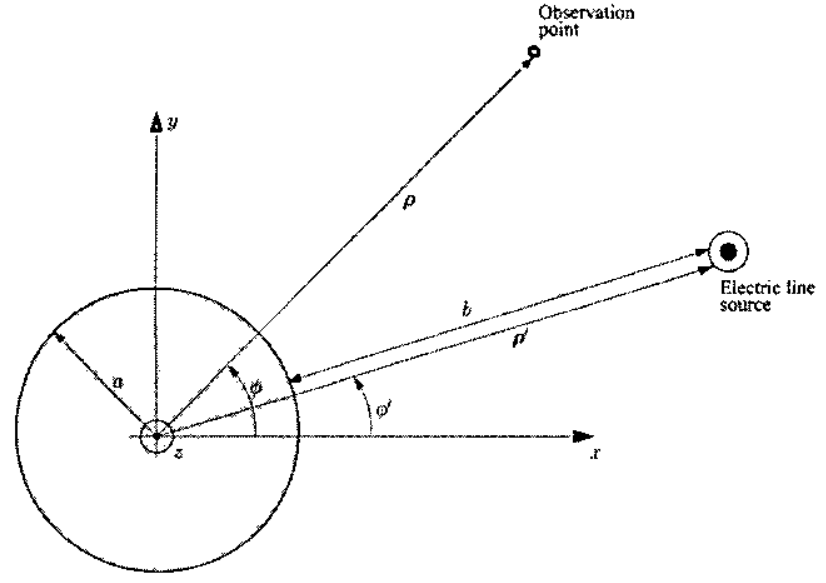

Figure 1. The antenna configuration: a DPS or DNG cylinder illuminated by an electric line source located in free space.

$\dot{\eta}_{0}=\sqrt{\mu_{0} / \varepsilon_{0}}$. The cylinder is of infinite extent, and has a radius denoted by $a$. The permittivity and the permeability of the cylinder are real scalars denoted by $\varepsilon_{c}=\varepsilon_{r} \varepsilon_{0}$ and $\mu_{c}=\mu_{r} \mu_{0}$, respectively, where $\varepsilon_{r}$ and $\mu_{r}$, are respectively its relative permittivity and permeability. The wavenumber inside the cylinder is denoted by $k_{c}$. For the DPS cylinder, $\varepsilon_{r}>0, \mu_{r}>0$, and $k_{c}=k_{0} \sqrt{\varepsilon_{r} \mu_{r}}>0$. For the DNG cylinder, $\varepsilon_{r}<0$ and $\mu_{r}<0$, and the sign of $k_{c}$ will be chosen in Section 2.3.

A cylindrical coordinate system $(\rho, \phi, z)$, with the associated Cartesian coordinate system $(x, y, z)$, is introduced such that the $z$ axis coincides with the cylinder's axis (see Figure 1). The position vector of a given observation point is $\rho$, and its coordinates are $(\rho, \phi)$, while the position vector of the electric line source is $\rho$, and its coordinates are $\left(\rho^{\prime}, \phi^{\prime}\right)$. The distance between the electric line source and the cylinder's surface is denoted by $b=\rho^{\prime}-a$.

\subsection{DPS Cylinders}

While the problem of plane-wave illumination of DPS cylinders has been thoroughly treated, see, e.g., the well-known textbook solutions [22, Chap. 17; 23, Chap. 6; 24, Chap. 11], the problem of the electric-line-source illumination is not as well documented in the literature. In [25, Chap. 3], however, a general procedure for finding the solution to the problem of electric-line-source illumination of DPS cylinders was given. The field expressions resulting from this procedure - which is based on an eigenfunction-expansion method - can be manipulated to obtain the explicit expressions for the problem at hand. The eigenfunction-expansion method was also applied in [24, Chap. 11] to derive the explicit expressions for perfectly electric conducting (PEC) cylinders in the presence of an electric line source, and this is easily extended to DPS cylinders, as shown below.

The incident field of the electric line source is $[24, p .573]$

$$
E^{i}(\rho)=-\hat{z} I_{e} \frac{\omega \mu_{0}}{4} H_{0}^{(2)}\left(k_{0}|\rho-\rho|\right)
$$

where $H_{0}^{(2)}(\cdot)$ is the Hankel function of second kind and zeroth 

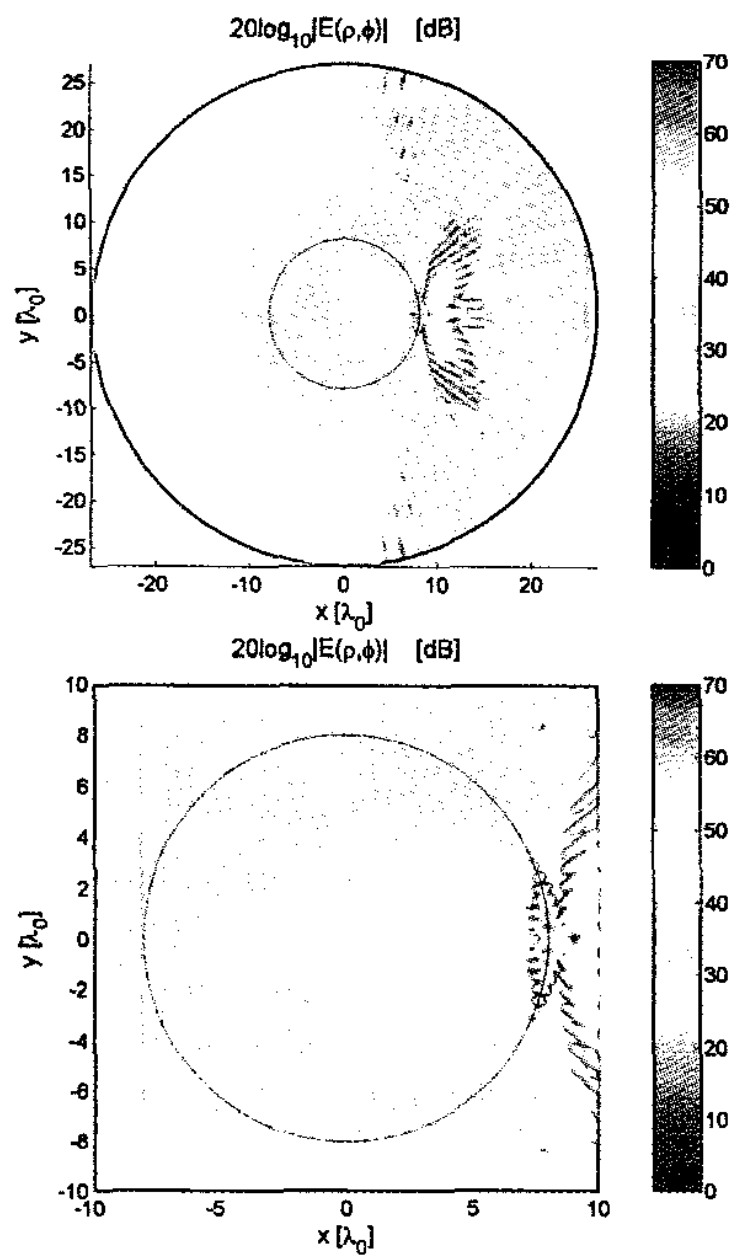

Figure 4. (top) The electric-field distribution for a " $1 \varepsilon_{0} 1 \mu_{0} "$ DNG cylinder having a radius of $a=8 \lambda_{0}$ with the electric line source at a distance $b=1 \lambda_{0}$ from the cylinder. (bottom) A selected region of the top plot, showing a closer look of the bigh-field-value formation.
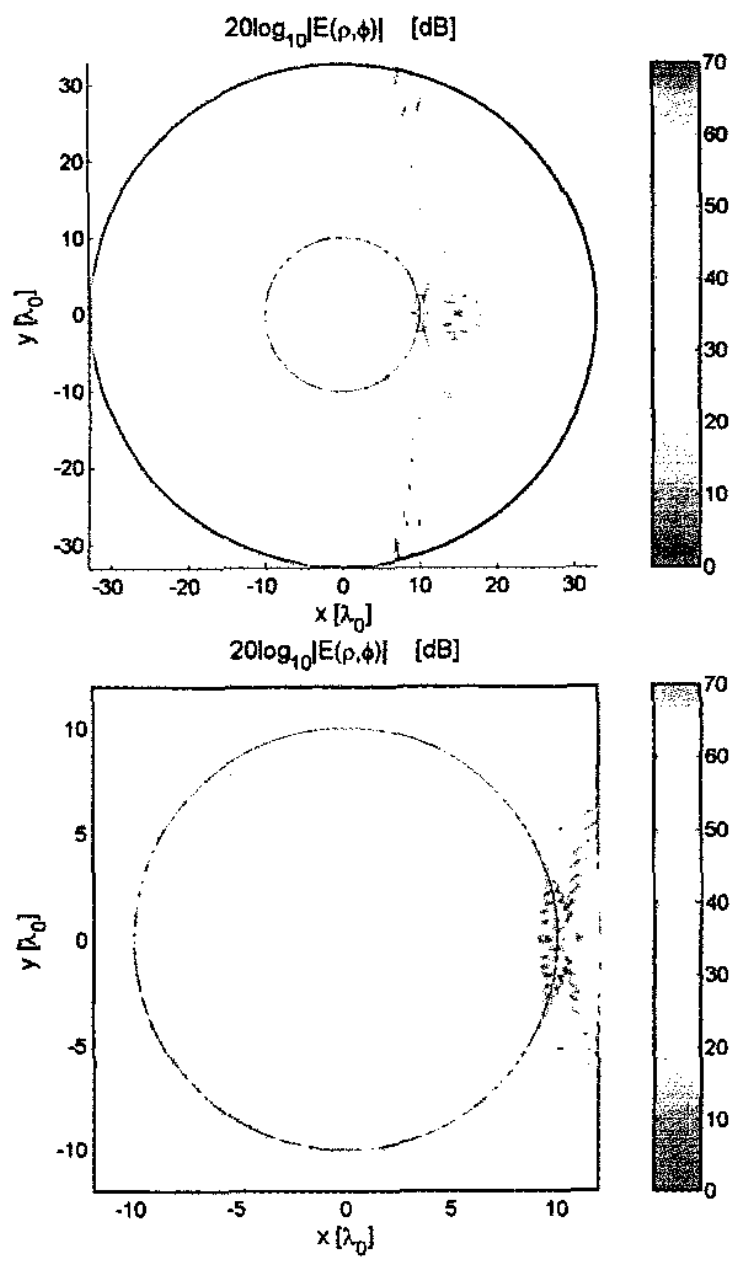

Figure 5. (top) The electric-field distribution for a " $1 \varepsilon_{0} l \mu_{0}$ " DNG cylinder baving a radius of $a=10 \lambda_{0}$ with the electric line source at a distance $b=1 \lambda_{0}$ from the cylinder. (bottom) A selected region of the top plot, showing a closer look of the high-field-value formation. 
order. Note that $\mathbf{E}^{i}$ in Equation (1) is given in terms of cylindrical wave functions that originate at the electric line source. In order to facilitate the forthcoming analysis, the incident electric field of Equation ( $l$ ) is expressed in terms of cylindrical wave functions that originate at the origin of the coordinate system, i.e., at $\rho=0$. Employing the addition theorem for Hankel functions, the incident electric field of the electric line source takes the form of $[24, p .626]$

$$
\begin{aligned}
& \mathbf{E}^{i}(\rho, \phi)= \\
& -i I_{e} \frac{\omega \mu_{0}}{4}\left\{\begin{array}{l}
\sum_{n=-\infty}^{\infty} J_{n}\left(k_{0} \rho\right) H_{n}^{(2)}\left(k_{0} \rho^{\prime}\right) e^{j n\left(\phi-\phi^{\prime}\right)} \text { for } \rho \leq \rho^{\prime} \\
\sum_{n=-\infty}^{\infty} J_{n}\left(k_{0} \rho^{\prime}\right) H_{n}^{(2)}\left(k_{0} \rho\right) e^{j n\left(\phi-\phi^{\prime}\right)} \text { for } \rho \geq \rho^{\prime}
\end{array}\right.
\end{aligned}
$$

The function $J_{n}(\cdot)$ is the Bessel function of order $n$, and is chosen to represent the field for $\rho \leq \rho^{\prime}$ due to its nonsingular behavior at the origin. $H_{n}^{(2)}(\cdot)$, which is the Hankel function of the second kind and order $n$, is chosen for $\rho \geq \rho^{\prime}$ due to its outward propagating nature, in compliance with the radiation condition. Similarly, the scattered and the transmitted electric fields, respectively, are expanded as

$$
\begin{aligned}
& E^{s}(\rho, \phi)=-\hat{z} I_{e} \frac{\omega \mu_{0}}{4} \sum_{n=-\infty}^{\infty} \psi_{n} H_{n}^{(2)}\left(k_{0} \rho\right) e^{j n\left(\phi-\phi^{\prime}\right)} \text { for } \rho \geq a \\
& \mathbf{E}^{i}(\rho, \phi)=-\hat{z} I_{e} \frac{\omega \mu_{0}}{4} \sum_{n=-\infty}^{\infty} \chi_{n} J_{n}\left(k_{c} \rho\right) e^{j n\left(\phi-\phi^{\prime}\right)} \text { for } \rho \leq a
\end{aligned}
$$

where $\psi_{n}$ and $\chi_{n}$ are the unknown expansion coefficients. It is noted that outside the cylinder, the total field is the sum of the incident, Equation (2), and scattered, Equation (3), fields, while inside the cylinder, the total field equals the transmitted field, Equation (4). The associated incident, scattered, and transmitted magnetic fields, which are required in the determination of the expansion coefficients, are readily obtained from Faraday's law. The electromagnetic-field boundary conditions, requiring the tangential components of the total electric and magnetic fields to be continuous at the cylinder's surface, are then applied. It follows that the expansion coefficients can be expressed as

$$
\psi_{n}=H_{n}^{(2)}\left(k_{0} \rho^{\prime}\right) \frac{J^{\prime}{ }_{n}\left(k_{0} a\right) J_{n}\left(k_{c} a\right)-\frac{\mu_{0} k_{c}}{\mu_{c} k_{0}} J_{n}\left(k_{0} a\right) J_{n}^{\prime}\left(k_{c} a\right)}{\frac{\mu_{0} k_{c}}{\mu_{c} k_{0}} J_{n}^{\prime}\left(k_{c} a\right) H_{n}^{(2)}\left(k_{0} a\right)-J_{n}\left(k_{c} a\right) H_{n}^{(2)}\left(k_{0} a\right)}
$$

and

$$
\chi_{n}=H_{n}^{(2)}\left(k_{0} \rho^{\prime}\right) \frac{J_{n}\left(k_{0} a\right) H_{n}^{(2)^{\prime}}\left(k_{0} a\right)-J_{n}^{\prime}\left(k_{0} a\right) H_{n}^{(2)}\left(k_{0} a\right)}{J_{n}\left(k_{c} a\right) H_{n}^{(2)^{\prime}}\left(k_{0} a\right)-\frac{\mu_{0} k_{c}}{\mu_{c} k_{0}} J_{n}^{\prime}\left(k_{c} a\right) H_{n}^{(2)}\left(k_{0} a\right)}
$$

It is noted that all derivatives in the above equations, indicated by primed symbols, are to be taken with respect to the function's entire argument.

As regards the practical implementations of the field expressions, it is first noted that it is convenient to fold the summation over $n$, employed in the exact solution, which ranges from $-\infty$ to $\infty$, to range from 0 to $\infty$ : For the scattered electric field given by Equation (3), it holds that

$$
\begin{aligned}
\mathbf{E}^{s}(\rho, \phi) & =-\hat{z} I_{e} \frac{\omega \mu_{0}}{4} \sum_{n=-\infty}^{\infty} \psi_{n} H_{n}^{(2)}\left(k_{0} \rho\right) e^{j n\left(\phi-\phi^{\prime}\right)} \\
& =-\hat{z} I_{e} \frac{\omega \mu_{0}}{4} \sum_{n=0}^{\infty} \varepsilon_{n} \psi_{n} H_{n}^{(2)}\left(k_{0} \rho\right) \cos \left[n\left(\phi-\phi^{\prime}\right)\right],
\end{aligned}
$$

where $\varepsilon_{n}$ is the Neumann number, which equals 1 for $n=0$, and 2 , otherwise. Similar transformations are employed for the remaining field expressions of Equations (2) and (4). Second, the summations in use must be truncated at some $n=N_{m a x}$, chosen in a manner that ensures convergence. Experience has shown that in general this is accomplished by selecting $N_{\max }=k_{0} \rho^{\prime}+20$ for all of the configurations treated in the present manuscript.

The far-field properties of the cylinders at hand are investigated through an examination of the two-dimensional directivity, henceforth referred to as simply the directivity, given by

$$
\begin{aligned}
& D(\phi)=\frac{2 \pi\left|\mathbf{E}_{f}^{\operatorname{tot}}(\phi)\right|^{2}}{\int_{\phi=0}^{2 \pi}\left|\mathbf{E}_{f}^{\operatorname{tot}}(\phi)\right|^{2} d \phi} \\
& =\frac{2 \pi\left|\mathbf{E}_{f}^{i}(\phi)+\mathbf{E}_{f}^{s}(\phi)\right|^{2}}{\int_{\phi=0}^{2 \pi}\left|\mathbf{E}_{f}^{i}(\phi)+\mathbf{E}_{f}^{s}(\phi)\right|^{2} d \phi}
\end{aligned}
$$

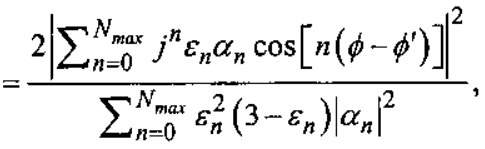

where $\alpha_{n}=J_{n}\left(k_{0} \rho^{\prime}\right)+\psi_{n}$, with $\psi_{n}$ given by Equation (5). In Equation (8), $\mathbf{E}_{f}^{\text {sot }}$ designates the total far-field pattern, which is the sum of the incident, $\mathbf{E}_{f}^{i}$, and the scattered, $\mathbf{E}_{f}^{s}$, far-field patterns.

These are easily obtained from Equation (2) (the expression for $\rho \geq \rho^{\prime}$ ) and Equation (3), respectively, through a large-argument expansion of the Hankel function involved [32, Chap. 10] and subsequent normalization with $\exp \left(-j k_{0} \rho\right) / \sqrt{\rho}$. In addition, the radiation resistance, $R_{r a d}^{\text {tot }}$, of the system consisting of a given cylinder and the electric line source is calculated and subsequently normalized to the radiation resistance, $R_{\text {rad }}^{i}$, due to the electric line source alone. This normalized radiation resistance is denoted by $R_{\text {rad }}^{\text {norm }}$, and is given by

$$
R_{r a d}^{n o r m}=\frac{R_{r a d}^{\text {tot }}}{R_{r a d}^{i}}=\frac{P_{r a d}^{1 o t}}{P_{r a d}^{i}}
$$

where

$$
P_{r a d}^{t o t}=\frac{1}{2 \eta_{0}} \int_{\phi=0}^{2 \pi}\left|\mathbf{E}_{f}^{t o t}(\phi)\right|^{2} d \phi=\frac{I_{e}^{2} k_{0} \eta_{0}}{16} \sum_{n=0}^{N_{\max }} \varepsilon_{n}^{2}\left(3-\varepsilon_{n}\right)\left|\alpha_{n}\right|^{2}
$$

is the power radiated by the system, and

$$
P_{r a d}^{i}=\frac{1}{2 \eta_{0}} \int_{\phi=0}^{2 \pi}\left|\mathbf{E}_{f}^{i}(\phi)\right|^{2} d \phi=\frac{I_{e}^{2} k_{0} \eta_{0}}{8}
$$



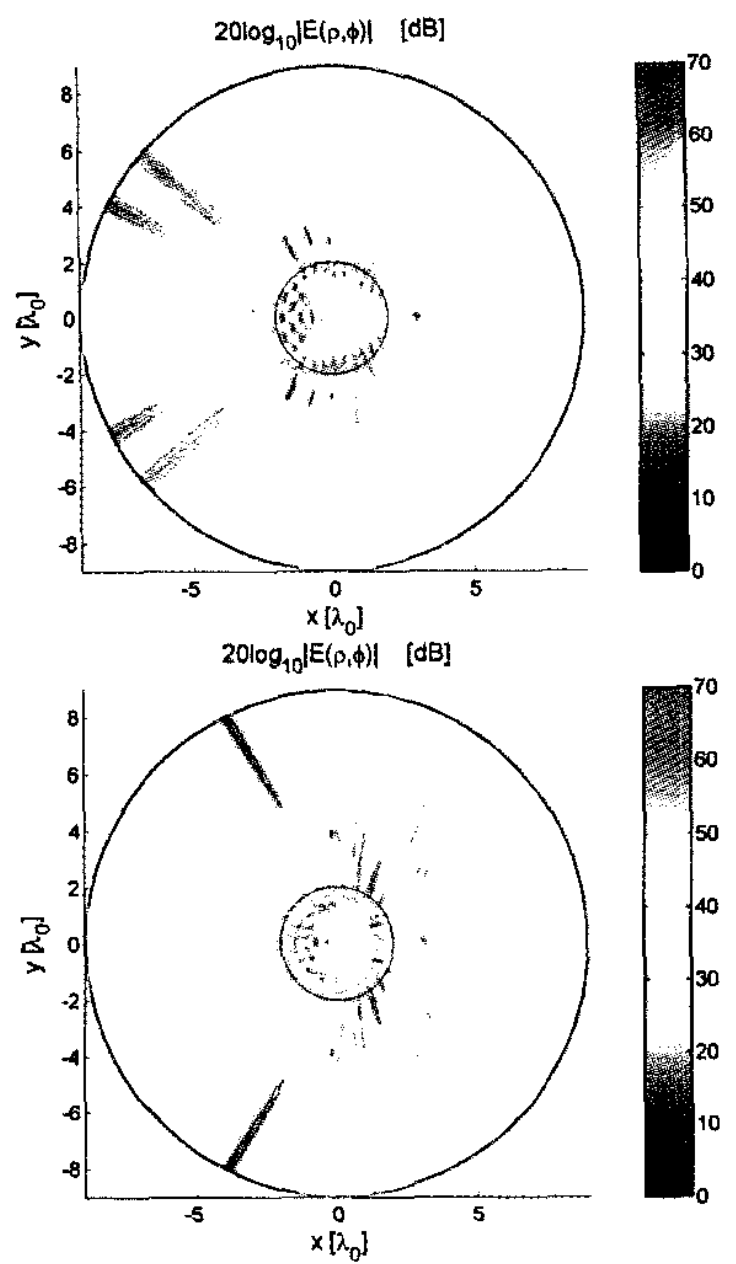

Figure 6. The electric-field distribution for " $2 \varepsilon_{0} 1 \mu_{0} "$ DPS (top) and DNG (bottom) cylinders having radii of $a=2 \lambda_{0}$. The electric line source was at a distance $b=1 \lambda_{0}$ from the cylinders.
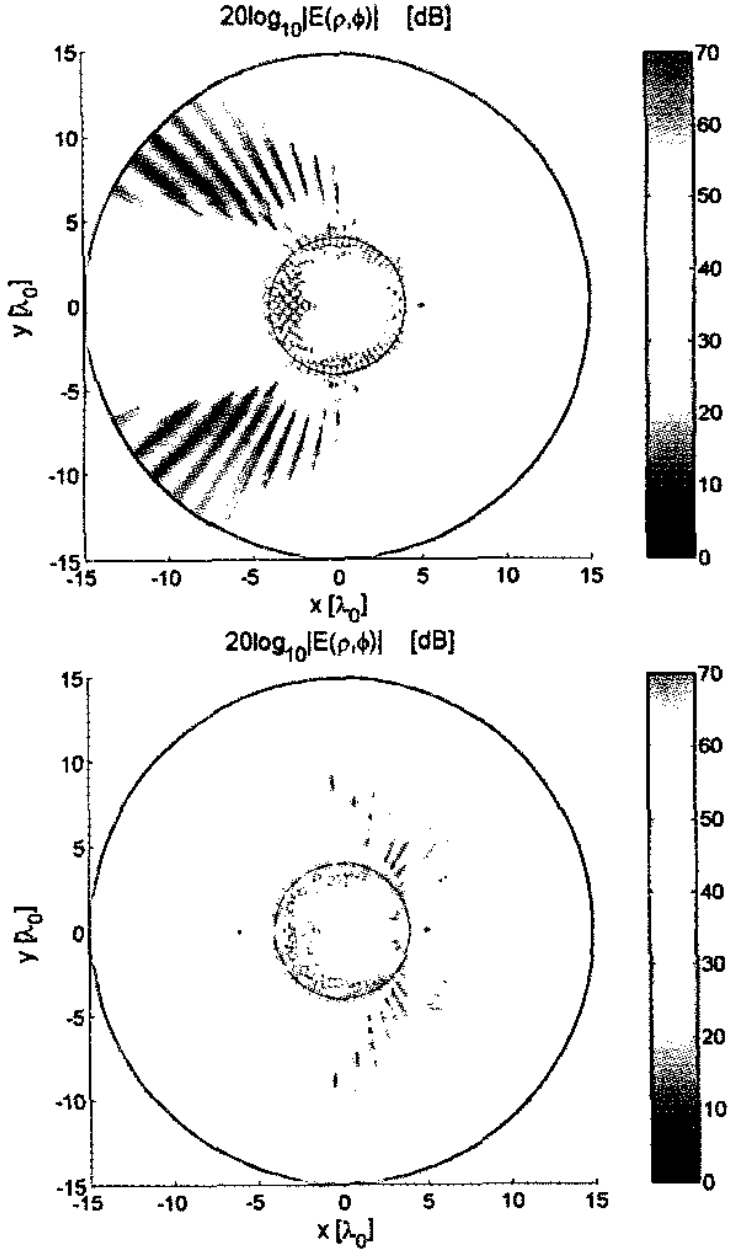

Figure 7. The electric-field distribution for " $2 \varepsilon_{0} 1 \mu_{0}$ " DPS (top) and DNG (bottom) cylinders having radii of $a=4 \lambda_{0}$. The electric line source was at a distance $b=1 \lambda_{0}$ from the cylinders. 
is the power radiated by the electric line source, alone.

\subsection{DNG Cylinders}

Two different ways of obtaining the solution to the DNG cylinder problem are presented in the following. Since they essentially differ in the choice of signs for some important parameters for DNG materials, it is appropriate to briefly discuss the various choices of the signs as adopted in the literature.

The sign of such parameters as refractive index, wavenumber, and intrinsic impedance for DNG materials has been the subject of some discussion in the literature: see, e.g., $[1,6-9,12,26-30]$ for a few examples. Most of the works, including the pioneering work of Veselago [1], devote the majority of their discussion to the sign of the refractive index. In [1], it was argued that the refractive index must be negative in a DNG material in order to have a correct description of the plane-wave refraction process taking place at a plane interface separating a DPS and a DNG material. No explicit mentioning of the sign of the wavenumber and intrinsic impedance was made in [1]. The negative refractive index was later adopted, among others, by $[6-9,12,26,27]$. In [27], the negative refractive index implied also a negative wavenumber, and it was moreover argued that a positive intrinsic impedance of a DNG material must be used. The latter was also explicitly stated in $[9,12,26]$. These findings were subsequently used to solve the problem of a DNG spherical shell surrounding an electrically small antenna [18] by simply using a negative wavenumber and a positive intrinsic impedance in the familiar expressions for the DPS spherical shell. Hence, in accordance with [27], the DNG cylinder solution can be obtained from the DPS cylinder solution derived in the previous section simply by inserting $\varepsilon_{c}<0, \mu_{c}<0$, and $k_{c}<0$ at appropriate places in Equations (1)-(10).

However, other works argue that a positive sign of the refractive index in a DNG material can likewise be used: see, e.g., [28-31]. By considering plane-wave propagation in an unbounded DNG material, [28] showed that the refractive index could be assumed positive if the unit propagation vector is defined in such a manner that it forms a left-handed set of vectors with the electric and magnetic field intensity vectors. In addition, [30, 31$]$ have shown that in unbounded DNG materials, the sign of the wavenumber merely depends on the choice of the partial solution to the wave equation, and that for either choice of the sign of the wavenumber, the intrinsic impedance and the refractive index can be defined positive as well as negative. Regarding the problem of scattering from DPS and DNG cylinders, $[30,31]$ have moreover shown that the eigenfunction solution, Equations (1)-(6), applies to DPS as well as DNG cylinders. For the DNG cylinder, one can choose $\varepsilon_{r}<0, \mu_{r}<0$, and $k_{c}>0$, on the one hand, or $\varepsilon_{r}<0$, $\mu_{r}<0$, and $k_{c}<0$, on the other hand, and the two choices give the same result. This is easily verified by using the analytic continuation formula for the Bessel function of order $n$ and negative argument, say $-x$ with $x>0[32$, p. 361$]$,

$$
J_{n}(-x)=(-1)^{n} J_{n}(x)
$$

as well as the relation

$$
J_{n}^{\prime}(-x)=-(-1)^{n} J_{n}^{\prime}(x)
$$

at appropriate places in Equations (1)-(10).
In summary, the results for the DNG cylinders are obtained from Equations (1)-(10) with $\varepsilon_{r}<0$ and $\mu_{r}<0$, and with either positive or negative $k_{c}$.

\section{Numerical Results for DPS and DNG Cylinders}

The numerical calculations were performed for different geometrical and electromagnetic parameters, such as cylinder radius, $a$; electric-line-source position, $b$; cylinder permittivity, $\varepsilon_{c}$; and permeability, $\mu_{c}$ (see Figure 1). In all of the forthcoming calculations, the coordinate system was aligned in such a manner that the electric line source was placed along the positive $x$ axis at a distance $b$ from the cylinder (see Figure 1). For easy reference, a cylinder having the material parameters $\left(\varepsilon_{c}, \mu_{c}\right)=\left(\varepsilon_{r} \varepsilon_{0}, \mu_{r} \mu_{0}\right)$ is referred to as an " $\varepsilon_{r} \varepsilon_{0} \mu_{r} \mu_{0}$ " DPS cylinder for $\varepsilon_{r}>0, \mu_{r}>0$, and as an " $\left|\varepsilon_{r}\right| \varepsilon_{0}\left|\mu_{r}\right| \mu_{0}$ " DNG cylinder for $\varepsilon_{r}<0, \mu_{r}<0$. (That is, a " $2 \varepsilon_{0} 1 \mu_{0}$ " DPS cylinder is a cylinder for which $\varepsilon_{r}=2$ and $\mu_{r}=1$, while a " $2 \varepsilon_{0} 1 \mu_{0}$ " DNG cylinder is a cylinder for which $\varepsilon_{r}=-2$ and $\mu_{r}=-1$.)

\subsection{Near-Field Properties}

First, the near-field properties of DNG cylinders were investigated. This was done by calculating the total field, denoted by $\mathbf{E}(\rho, \phi)$ inside as well as outside the cylinder. More specifically, the quantity $20 \log _{10}|\mathbf{E}(\rho, \phi)|[\mathrm{dB}]^{2}$ is shown in a circular region of radius $3 \rho^{\prime}$ centered at the cylinder's axis ( $z$ axis) (see Figure 1 ). To facilitate comparison of the results, the dynamic range was kept constant throughout the forthcoming figures.

\subsubsection{Results for " $1 \varepsilon_{0} 1 \mu_{0}$ " DNG cylinders}

The effect of the DNG material is particularly notable in the case of a " $1 \varepsilon_{0} l \mu_{0}$ " cylinder. A few examples of such results are shown in Figures 2 through 5, which depict the near-field distributions for the cylinders having radii $2 \lambda_{0}$ through $10 \lambda_{0}$, respectively. For all configurations, the electric line source was located at a distance $b=1 \lambda_{0}$ from the cylinder.

The " $1 \varepsilon_{0} 1 \mu_{0}$ " DPS cylinder was clearly equivalent to the free-space case without any scattering of the electric-line-source field, which is thus omnidirectional. In comparison to this, of course the corresponding " $1 \varepsilon_{0} l \mu_{0}$ " DNG cylinder had a significant effect on the field of the electric line source by creating a field pattern with several lobes and a shadow region. It was observed that the variation of the field pattern was naturally more pronounced for the cylinders having larger radii. In all cases, there appeared to be a formation of a point inside the cylinders at which the field assumed very high values. A similar effect was observed in [16] for the case of plane-wave incidence. This formation, though less clear for the cylinder of radius $2 \lambda_{0}$ than for the cylinders of larger radii, suggests that some focusing of the electric-line-source field took 

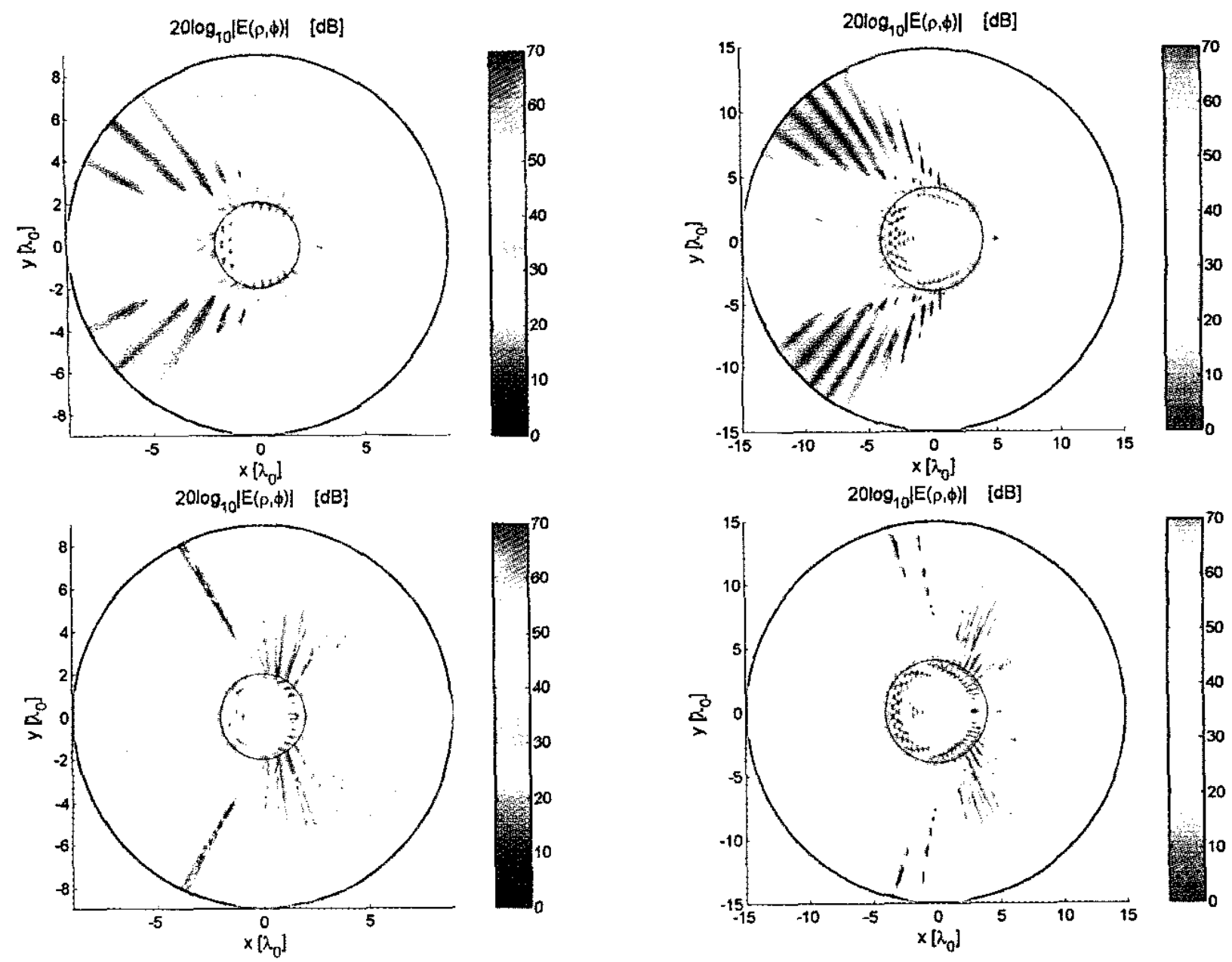

Figure 8. The electric-field distribution for " $1 \varepsilon_{0} 2 \mu_{0}$ " DPS (fop) and DNG (bottom) cylinders having radii of $a=2 \lambda_{0}$. The electric line source was at a distance $b=1 \lambda_{0}$ from the cylinders.

Figure 9. The electric-field distribution for " $1 \varepsilon_{0} 2 \mu_{0}$ "DPS (top) and DNG (bottom) cylinders having radii of $a=4 \lambda_{0}$. The electric line source was at a distance $b=1 \lambda_{0}$ from the cylinders. 
place inside the cylinders. Contrary to the well-known case of the lossiess DNG slab configuration of the same material parameters see, e.g., [1] and [12] - no high field values were observed in the region to the left of the cylinders along the negative $x$ axis. Moreover, the point inside the cylinders at which the field attained a high value did not represent an exact image of the electric line source, since the cylinder surface was curved and its aperture was finite. In addition to the bigh-field-value formation inside the cylinders, the field also attained rather high values along the part of the cylinder's surface that was close to the electric line source: see Figures $3 b, 4 b$, and $5 b$.

\subsubsection{Results for " $2 \varepsilon_{0} 1 \mu_{0}$ " and " $1 \varepsilon_{0} 2 \mu_{0}$ " DPS and DNG Cylinders}

Figures 6a and 7a show the field for " $2 \varepsilon_{0} 1 \mu_{0}$ " DPS cylinders having radii of $a=2 \lambda_{0}$ and $a=4 \lambda_{0}$, respectively, while Figures $8 \mathrm{a}$ and $9 \mathrm{a}$ show the field for " $1 \varepsilon_{0} 2 \mu_{0}$ " DPS cylinders having the same radii, respectively. In all cases, the electric line source was located at $b=1 \lambda_{0}$. The results for the corresponding DNG cylinders are shown in Figures $6 \mathrm{~b}-7 \mathrm{~b}$, and $8 \mathrm{~b}-9 \mathrm{~b}$, respectively.

For the DPS cylinders, a clear beam formation along the negative $x$ axis was observed, the beam being somewhat broader for the cylinders of larger radii. That is, the DPS cylinder configuration constituted a lens-like antenna. Some field variation was observed in the half-space in which the electric line source was located, and no shadow region was formed. For the DNG-cylinder configurations, the field pattern exhibited rather large azimuthal variation, the variations being more pronounced for the cylinders of larger radii. The field outside the DNG cylinders was mainly confined to the half space in which the electric line source was located. Furthermore, the field variation was more pronounced for the DNG than for the DPS cylinders in the sense that much deeper zeros were observed in the pattem. Regarding the field transmitted into the DNG cylinders, a clear formation of a high field value was observed, this being more clear than with the previously shown results for " $1 \varepsilon_{0} 1 \mu_{0}$ " DNG cylinders, again suggesting that some focusing of the electric-line-source field was taking place. For both DPS and DNG cylinders, the field inside them was higher for the cylinders possessing the larger $\mu_{c}$.

When the distance between the electric line source and the cylinder was increased to $b=2 \lambda_{0}$, the field variation increased notably, and, in the case of DNG cylinders, the formation of a high field value inside the cylinders was not as clear as with the $1 \lambda_{0}$ distance considered previously. This is illustrated in Figures $10 \mathrm{a}$ and $10 \mathrm{~b}$, which respectively show the results for " $2 \varepsilon_{0} 1 \mu_{0}$ " and " $1 \varepsilon_{0} 2 \mu_{0}$ " DNG cylinders having radii of $a=2 \lambda_{0}$ and the electric line source located at $b=2 \lambda_{0}$. If the distance between the cylinder and the electric line source was further increased, the field variation increased even further, and the high-field-value formation became very difficult to observe.

It is noted that DPS and DNG cylinders with various other combinations of the material parameters, investigated in our study but not included in this manuscript, gave rise to the same observations as made above.

In what follows, some results for DPS and DNG cylinders having a fairly small radius are shown. Figures $11 \mathrm{a}$ and $12 \mathrm{a}$ respec- tively show the near-field distribution for " $2 \varepsilon_{0} 1 \mu_{0}$ " and " $1 \varepsilon_{0} 2 \mu_{0}$ " DPS cylinders, while Figures $11 \mathrm{~b}$ and $12 \mathrm{~b}$ show the same results for the corresponding DNG cylinders. In all cases, the cylinder radius was $a=0.1 \lambda_{0}$, and the electric line source was $0.3 \lambda_{0}$ away from the cylinder's surface. As can be observed, the field patterns for DPS cylinders were very similar, while those for DNG cylinders differed considerably. In particular, very high field values were observed along the entire surface of the " $2 \varepsilon_{0} 1 \mu_{0}$ " DNG cylinder, while this was not the case for the " $1 \varepsilon_{0} 2 \mu_{0}$ " DNG cylinder. It was noted that the DNG cylinders had a much larger effect on the electric-line-source field than the corresponding DPS cylinders.

\subsubsection{Results for Different Electric Line Source Positions}

In the previous sections, it was shown that the field pattern outside the DNG cylinders exhibited a significant variation with the azimuthal direction of observation for a fixed position of the electric line source. Next, the variation of the field pattern with different positions of the electric line source is investigated to study the constructive/destructive interference of the incident and scattered fields.
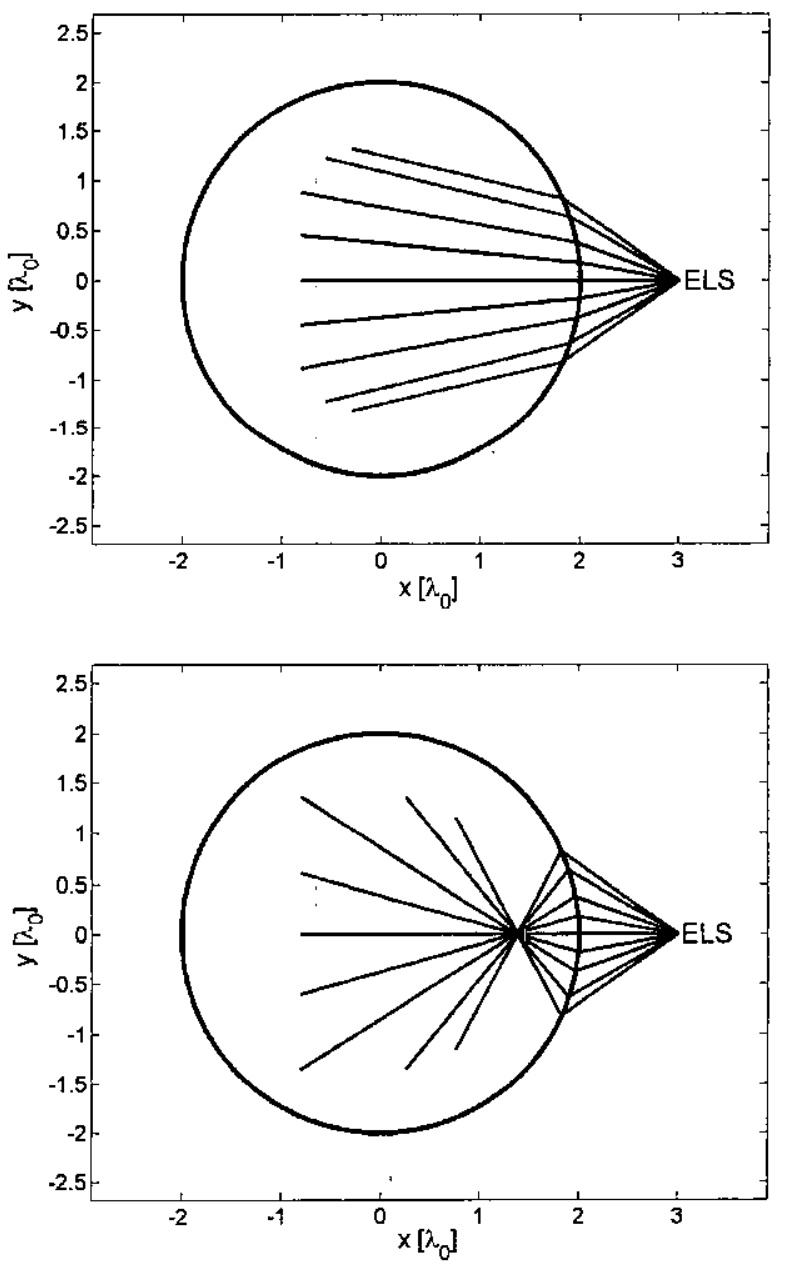

Figure 14. The GO ray-tracing results for " $2 \varepsilon_{0} 1 \mu_{0}$ " DPS (top) and DNG (bottom) cylinders having radii of $a=2 \lambda_{0}$. The electric line source was at a distance $b=1 \lambda_{0}$ from the cylinders. The corresponding eigenfunction solution is shown in Figure 6. 

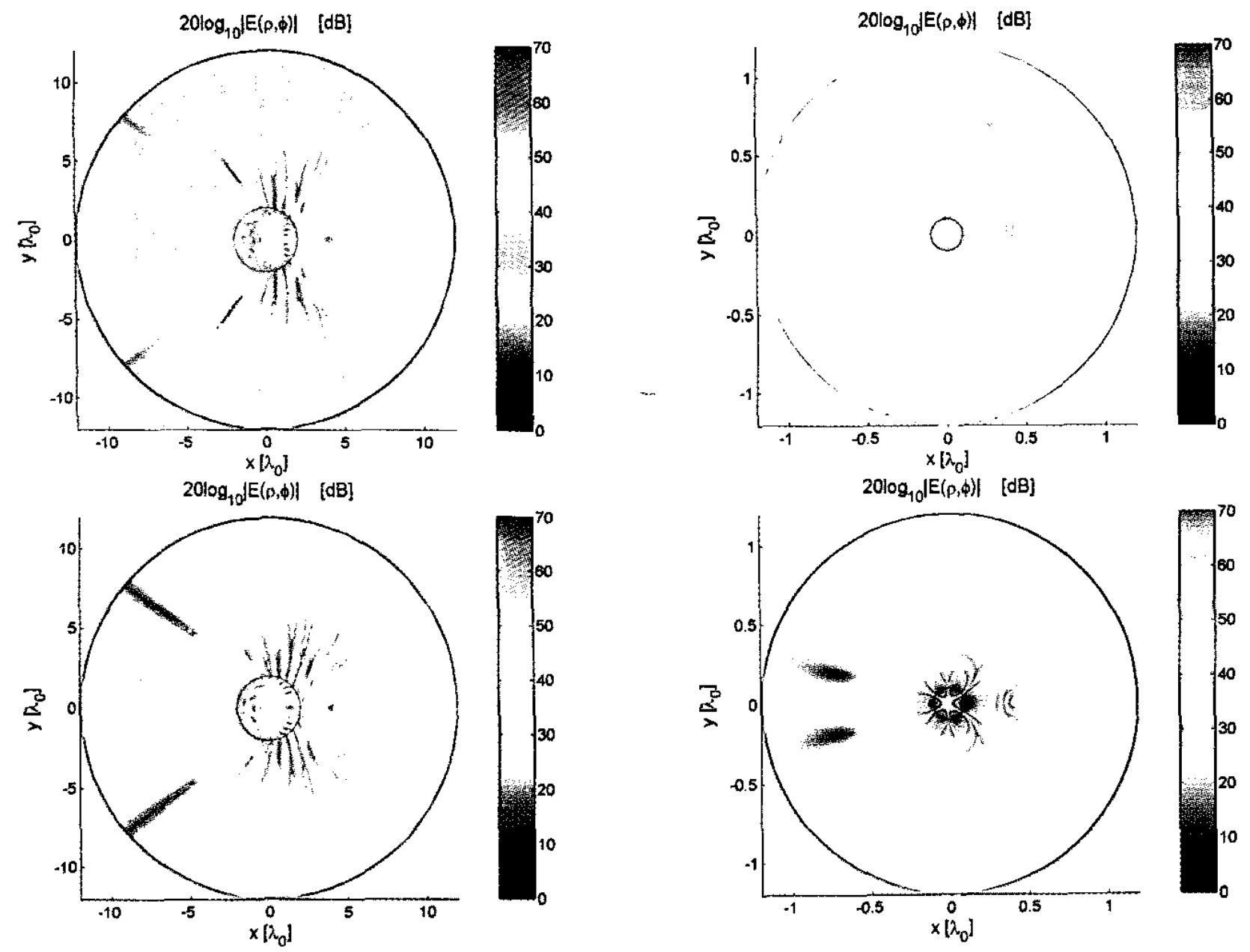

Figure 10. The electric-field distribution for " $2 \varepsilon_{0} 1 \mu_{0}$ " (top) and " $1 \varepsilon_{0} 2 \mu_{0}$ " (bottom) DNG cylinders having radii of $a=2 \lambda_{0}$. The electric line source was at a distance $b=2 \lambda_{0}$ from the cylinders.

Figure 11. The electric-field distribution for " $2 \varepsilon_{0} 1 \mu_{0}$ " DPS (top) and DNG (bottom) cylinders having radii of $a=0.1 \lambda_{0}$. The electric line source was at a distance $b=0.3 \lambda_{0}$ from the cylinders. 

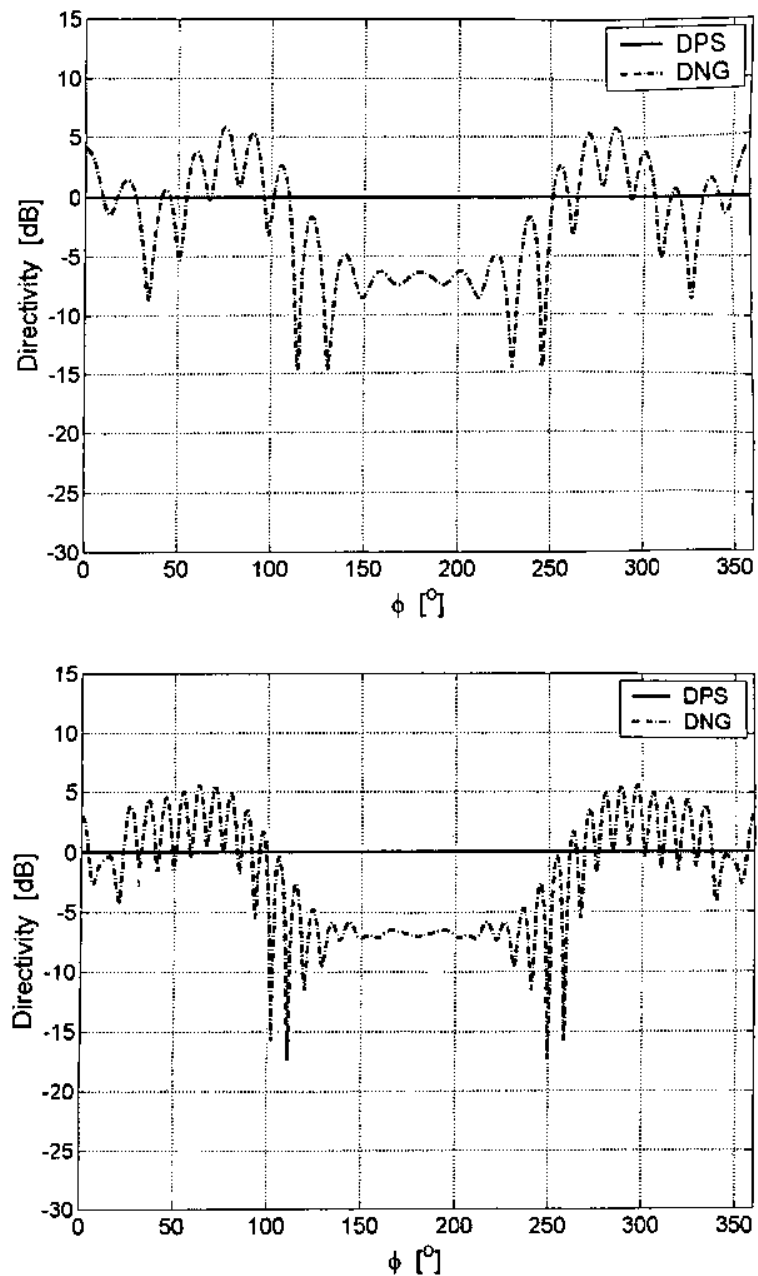

Figure 15. The directivity for " $1 \varepsilon_{0} 1 \mu_{0} " \mathrm{DPS}$ and DNG cylinders having radii of $a=2 \lambda_{0}$ (top) and $a=4 \lambda_{0}$ (bottom). The electric line source was at a distance $b=1 \lambda_{0}$ from the cylinders.

To this end, the field patterns were recorded for a " $1 \varepsilon_{0} 1 \mu_{0}$ " DNG cylinder of radius $a=2 \lambda_{0}$ and electric-line-source distances $b=[0.25,0.40,0.65,0.75] \lambda_{0}^{3}$ (see Figure 13). (The field patterns were obtained for other distances, as well. These were $b=[0.30$, $0.35,0.45,0.50,0.55,0.60,0.70] \lambda_{0}$. However, the effects explained in terms of constructive and destructive interferences were most in evidence for the distances $b=[0.25,0.40,0.65,0.75] \lambda_{0}$. Therefore, only the results for the latter distances are included here.)

It was observed that the patterns shown in Figures 13a and $13 \mathrm{~b}$, apart from being somewhat ripple-like, contained a sort of a main beam in the $\phi=0^{\circ}$ direction. This could be explained by the constructive interference taking place in these two cases. However, the main beam was more clear for the distance $b=0.40 \lambda_{0}$ than for the $b=0.25 \lambda_{0}$ : distance. In the case of the $b=0.65 \lambda_{0}$ distance (see Figure 13c), the main beam was completely absent, and this could be explained by the destructive interference. At the distance $b=0.75 \lambda_{0}$, the field began to attain high field values in the $\phi=0^{\circ}$ direction. However, due to a longer distance to the electric line source, this formation did not merge into any sort of a main beam, and it was not nearly as pronounced as in the case of the two smaller distances giving rise to the constructive interference (see Figures $13 \mathrm{a}$ and $13 \mathrm{~b}$ ). In addition, considerable sidelobes were present in the pattern of Figure 13d.

Similar investigations as above were performed for the " $2 \varepsilon_{0} 1 \mu_{0}$ " and " $1 \varepsilon_{0} 2 \mu_{0}$ " DNG cylinders. The effects of constructive and destructive interference were observed, but they were far from being as pronounced as for the case of " $1 \varepsilon_{0} 1 \mu_{0}$ " DNG cylinders shown here.

\subsubsection{Results in Terms of Geometrical-Optics Ray-Tracing Techniques}

It is interesting to note that the numerical results based on the eigenfunction-solution technique presented here can be interpreted by means of the Geometrical Optics (GO) ray-tracing technique.

A number of incident rays launched from the electric line source and forming angles $\alpha= \pm\left[0^{\circ}, 10^{\circ}, 20^{\circ}, 30^{\circ}, 35^{\circ}\right]$ with the $x$ axis were traced to their intersection points on the surface of the
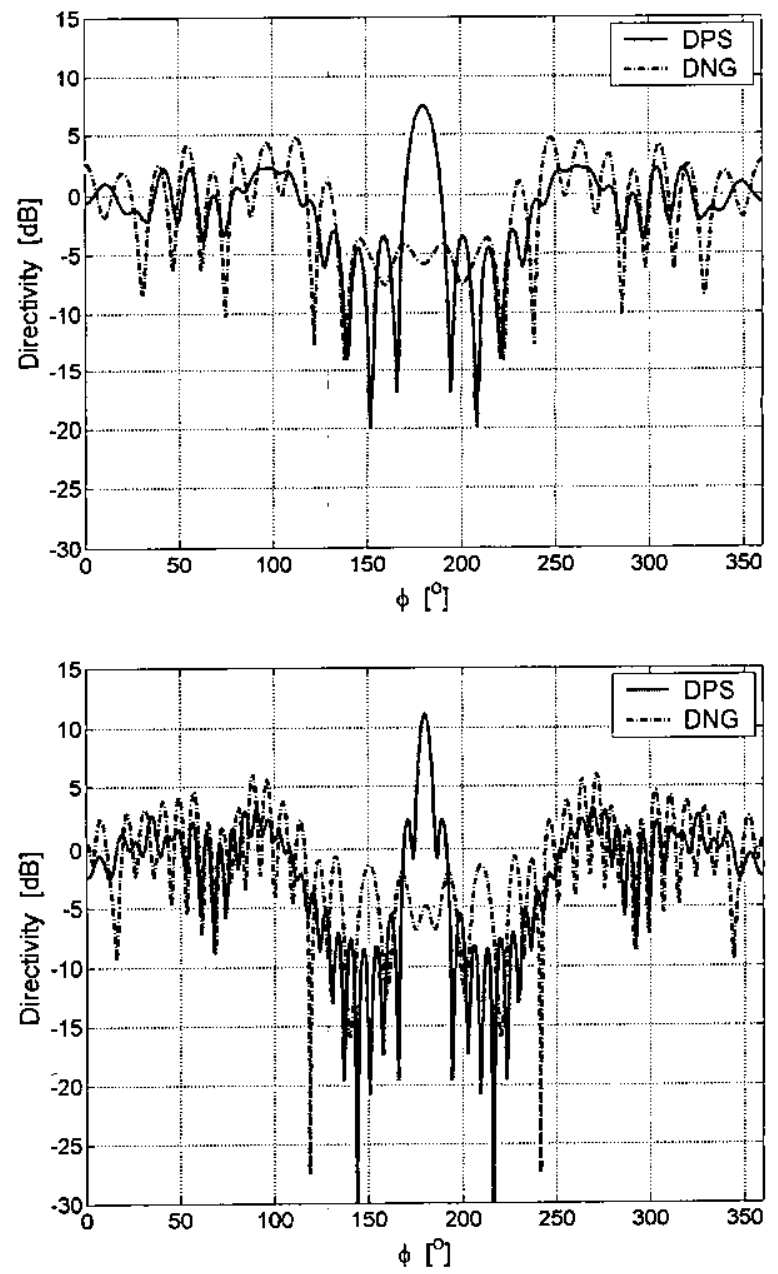

Figure 16. The directivity for " $2 \varepsilon_{0} 1 \mu_{0}$ " DPS and DNG cylinders baving radii of $a=2 \lambda_{0}$ (top) and $a=4 \lambda_{0}$ (bottom). The electric line source was at a distance $b=1 \lambda_{0}$ from the cylinders. 

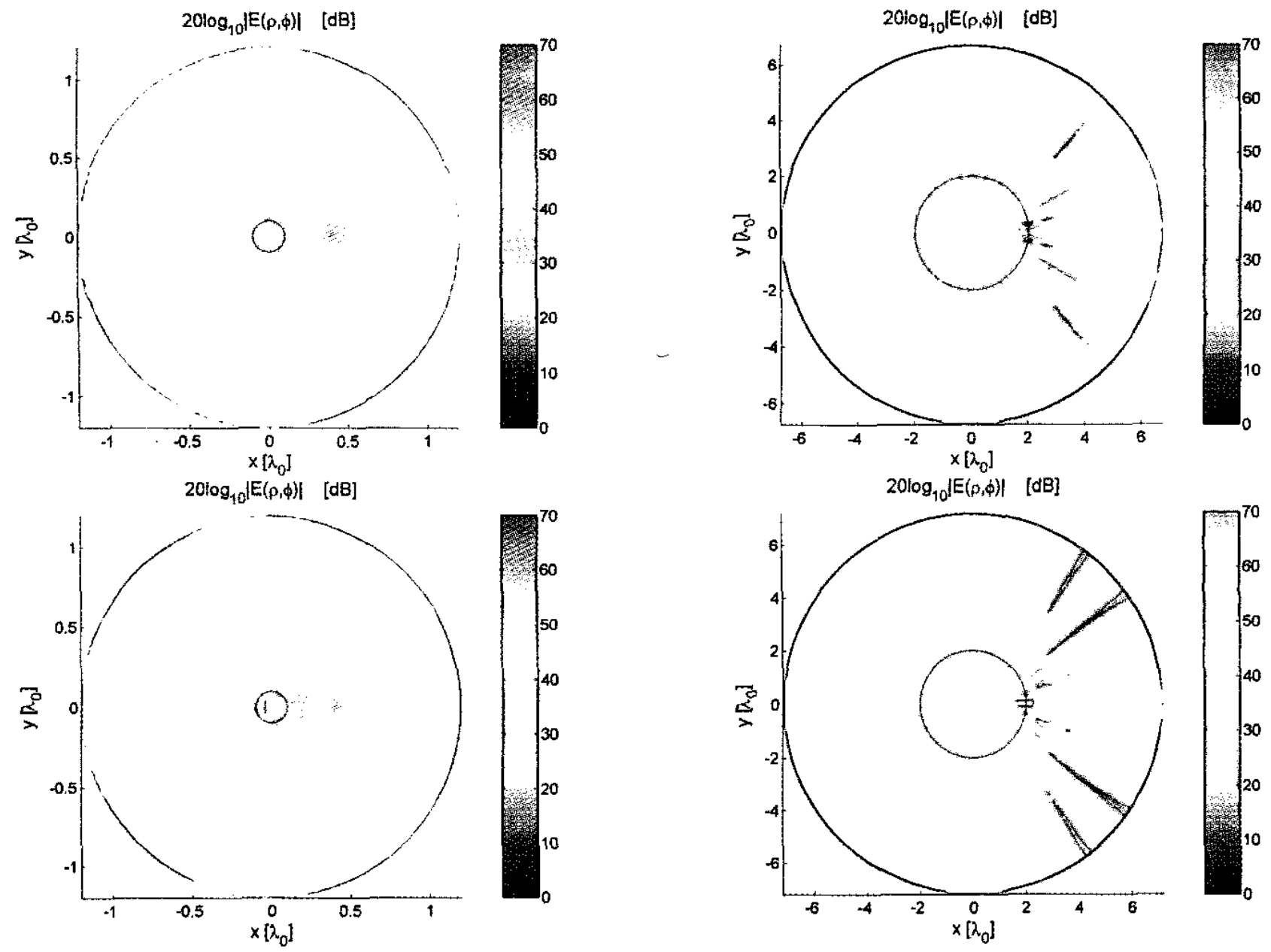

Figure 12. The electric-field distribution for " $1 \varepsilon_{0} 2 \mu_{0}$ " DPS (top) and DNG (bottom) cylinders having radii of $a=0.1 \lambda_{0}$. The electric line source was at a distance $b=0.3 \lambda_{0}$ from the cylinders.

Figure 13. The electric-field distribution for a " $1 \varepsilon_{0} 1 \mu_{0}$ " DNG cylinder having a radius of $a=2 \lambda_{0}$, with the electric line source placed at (top) $b=0.25 \lambda_{0}$, (second) $b=0.40 \lambda_{0}$, (third) $b=0.65 \lambda_{0}$, and (bottom) $b=0.75 \lambda_{0}$.
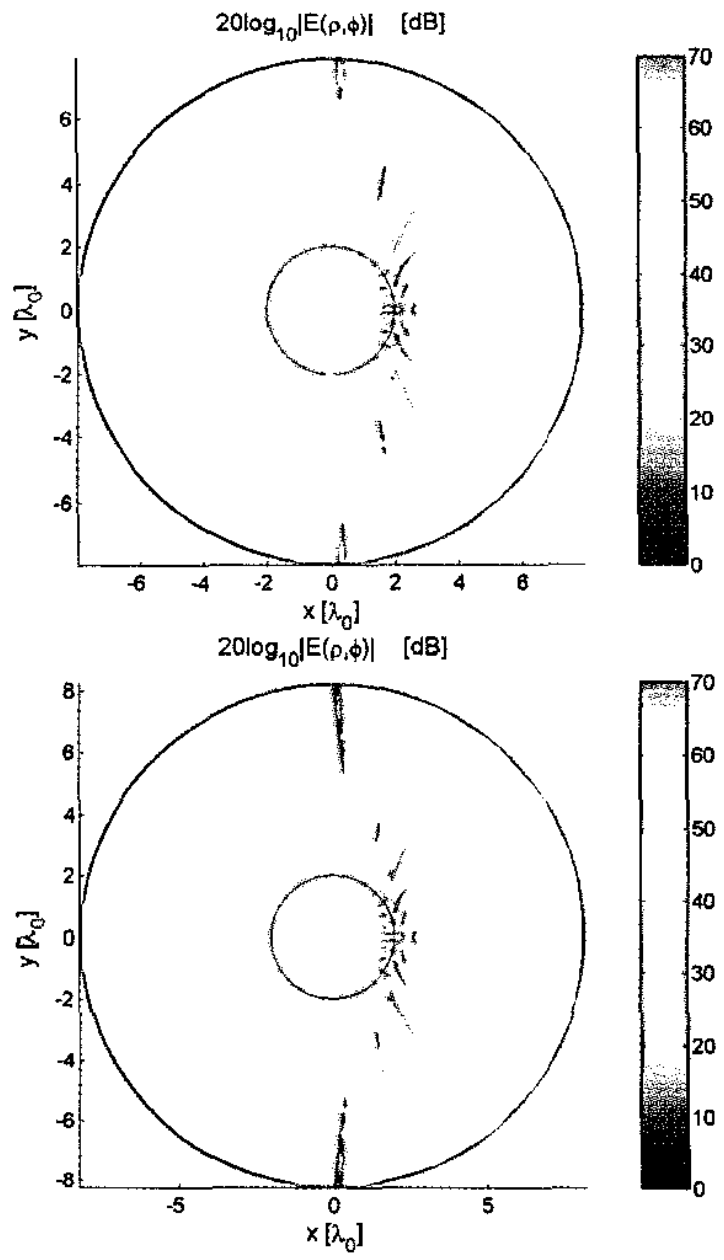

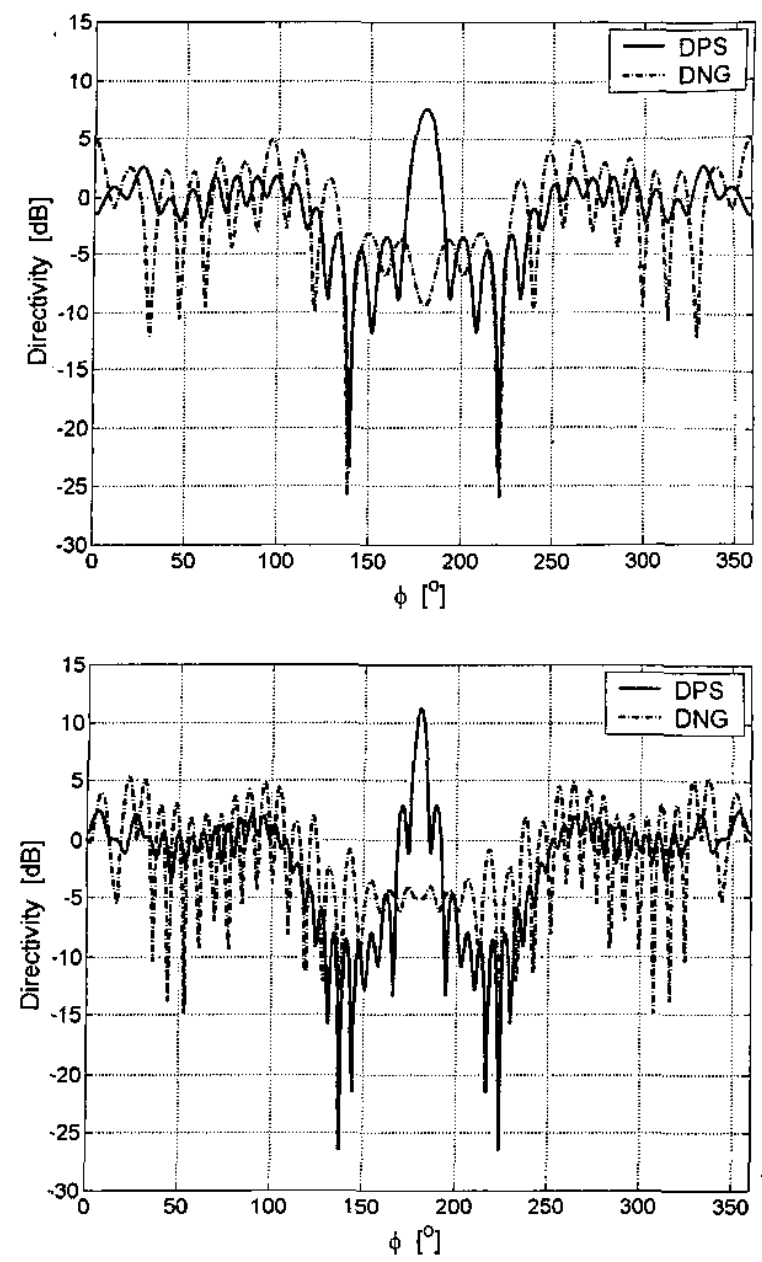

Figure 17. The directivity for " $1 \varepsilon_{0} 2 \mu_{0} "$ DPS and DNG cylinders having radii of $a=2 \lambda_{0}$ (top) and $a=4 \lambda_{0}$ (bottom). The electric line source was at a distance $b=1 \lambda_{0}$ from the cylinders.

cylinder. At these points, Snell's law of refraction was then used to determine the directions of the corresponding refracted rays into the cylinder.

As an example of these matters, the GO ray tracing was performed for the configuration for which the near fields are shown in Figure 6, and the result is shown in Figure 14.

Comparing the results shown in Figures 6 and 14, it was found that the GO ray tracing results supported the numerical results very well. The presence of a localized high field value for the DNG cylinder was also noted.

It was noted that the $\mathrm{GO}$ ray-tracing analysis was likewise performed, but not included here, for other configurations for which the near fields were previously shown. In all cases, the GO ray-tracing results supported the numerical results very well. For the case of plane-wave incidence, [16] reported a similar GO investigation.

\subsection{Far-Field Properties}

Second, the far-field properties of DNG cylinders were investigated. This was done by examining their directivity and the normalized radiation resistance, the analytical formulae for both of which were derived in Section 2.2.

\subsubsection{Directivity}

Figure 15 shows the directivity for the " $1 \varepsilon_{0} 1 \mu_{0}$ " DNG cylinder configuration for which the near fields were depicted in Figure 2. The directivity for the corresponding DPS cylinder is shown as well, and this was, of course, uniformly distributed. The DNG cylinders did not exhibit any main-lobe formation, and the pattern contained a number of ripples, this being more pronounced for the cylinder of the larger radius. These observations were in accordance with the near-field results shown in Figure 2. The formation of a shadow region was obvious in both cases, since the directivity attained much lower values for $\phi \in\left[125^{\circ}, 225^{\circ}\right]$ than for $\phi \in\left[0^{\circ}, 125^{\circ}[U] 225^{\circ}, 360^{\circ}[\right.$

Similar observations applied for cylinders of other material parameters. Figures $16 \mathrm{a}$ and $16 \mathrm{~b}$ show the directivity for DPS and DNG cylinders for which the near fields were shown in Figures 6 and 7 , respectively. In addition, Figures $17 \mathrm{a}$ and $17 \mathrm{~b}$ show the directivity for DPS and DNG cylinders for which the near fields were shown in Figures 8 and 9, respectively. In all cases, a clear lobe
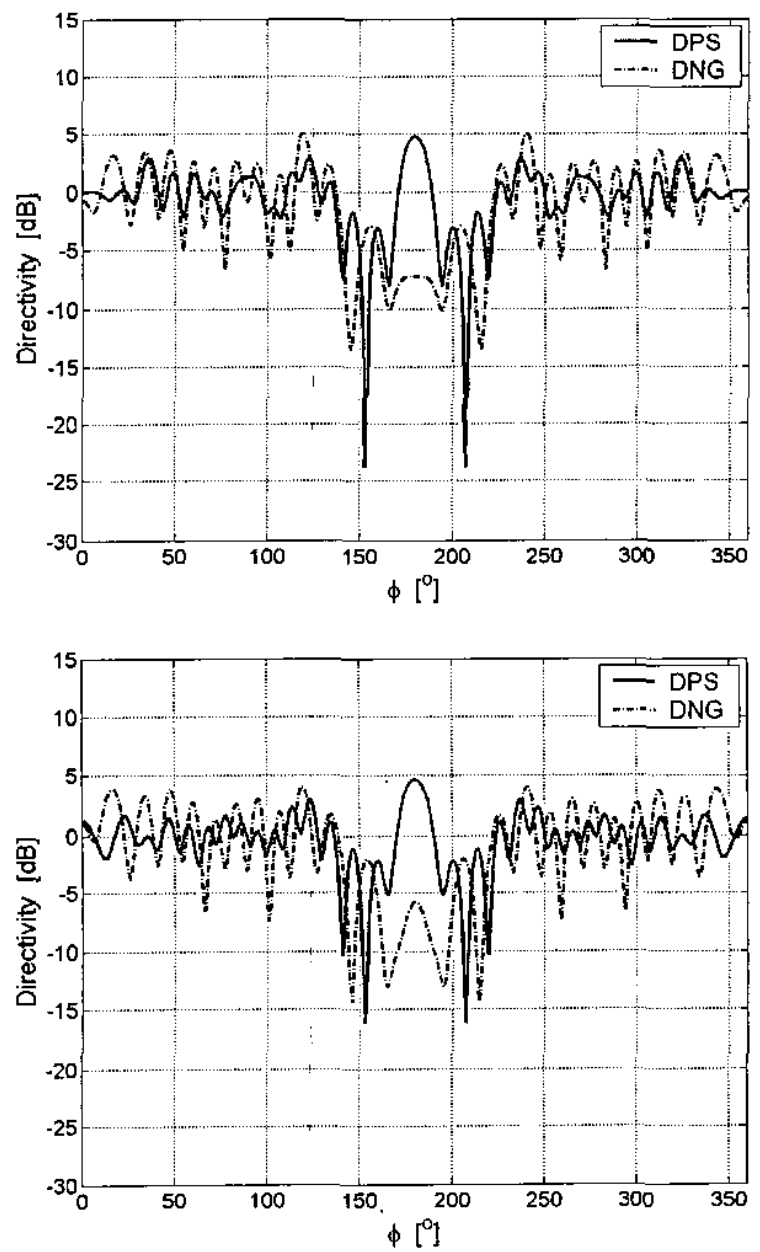

Figure 18. The directivity for " $2 \varepsilon_{0} 1 \mu_{0}$ " (top) and " $1 \varepsilon_{0} 2 \mu_{0}$ " (bottom) DPS and DNG cylinders having radii of $a=2 \lambda_{0}$. The electric line source was at a distance $b=2 \lambda_{0}$ from the cylinders. 

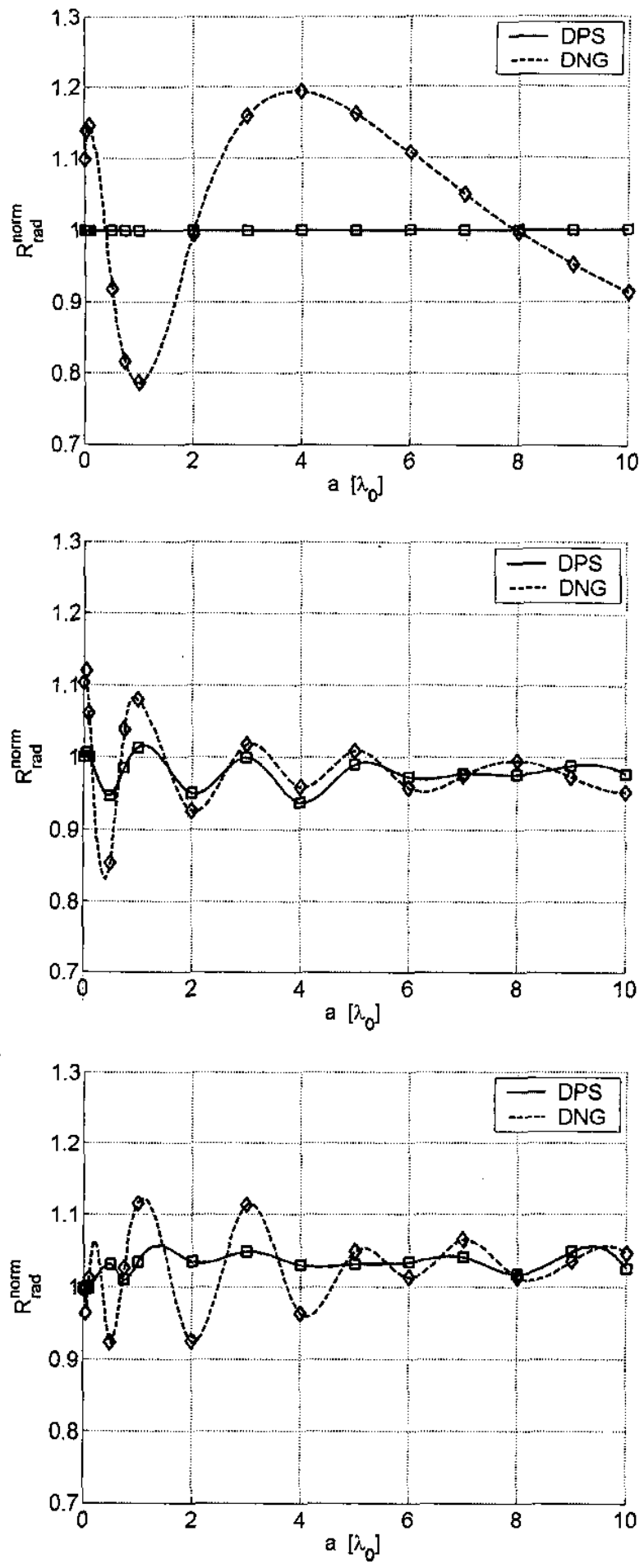

Figure 19. The variation of the normalized radiation resistance, $R_{\text {rad }}^{\text {norm }}$, as a function of the cylinder radius, $a$, for DPS and DNG cylinders of various material parameters: (top) " $1 \varepsilon_{0} 1 \mu_{0}$," (middle) " $2 \varepsilon_{0} 1 \mu_{0}$," (bottom) " $1 \varepsilon_{0} 2 \mu_{0}$ " The electric line source was at a distance $b=1 \lambda_{0}$ from the cylinders. The markers indicate those radit for which $R_{\text {rad }}^{\text {norm }}$ was calculated.
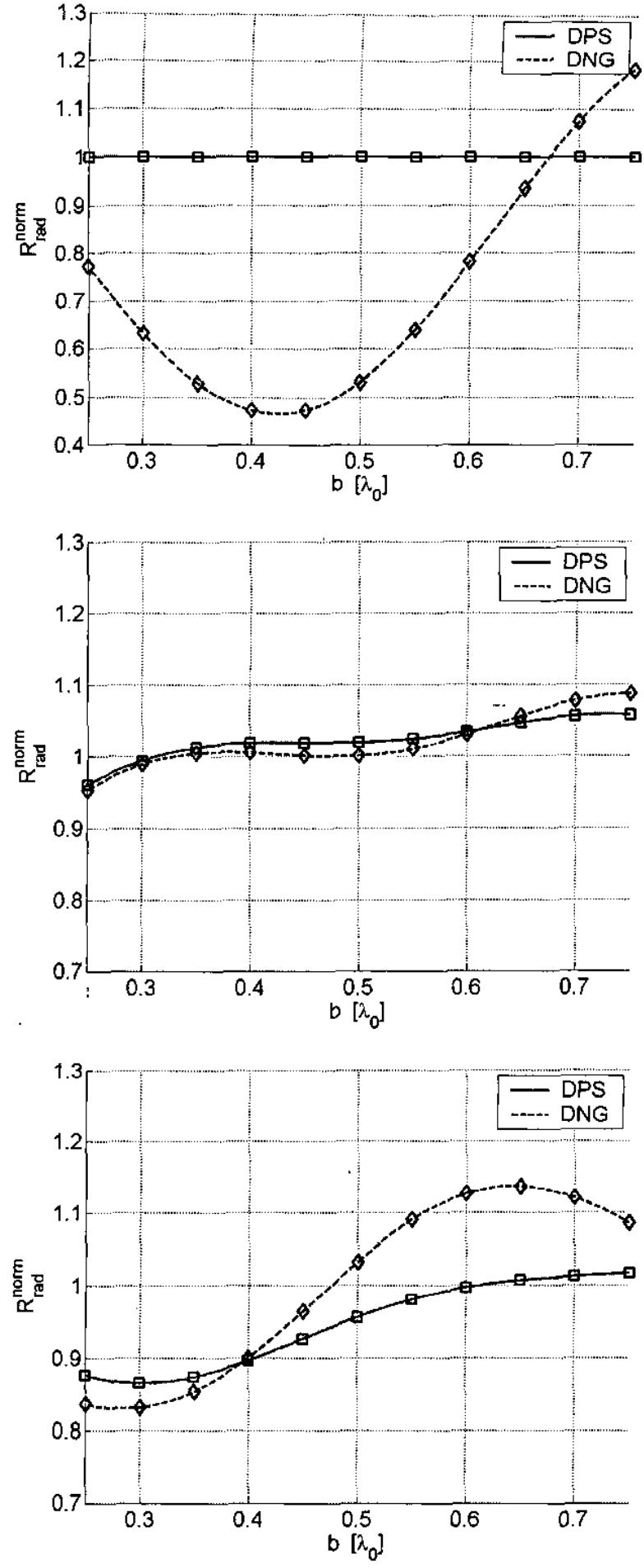

Figure 20. The variation of the normalized radiation resistance, $R_{\text {rad }}^{\text {norm }}$, as a function of the distance to the cylinder, $b$, for DPS and DNG cylinders of radius $a=2 \lambda_{0}$ and various material parameters: (top) " $1 \varepsilon_{0} 1 \mu_{0}, "$ (middle) " $2 \varepsilon_{0} l \mu_{0}$," (bottom) " $1 \varepsilon_{0} 2 \mu_{0}$." The markers indicate those radii for which $R_{\text {rad }}^{\text {norm }}$ was calculated. 
formation was observed for DPS cylinders in the angular region close to the $\phi=180^{\circ}$ direction. As the observation point was moved away from this direction, the pattern became ripple-like, this again being more pronounced for the larger cylinder. The DNG cylinder directivity was observed to contain no distinct main lobe, but instead a number of ripples in the pattern. In these cases, the shadow region around $\phi=180^{\circ}$ was easily identified. As illustrated in Figure 18 , this also held for the " $2 \varepsilon_{0} 1 \mu_{0}$ " and " $1 \varepsilon_{0} 2 \mu_{0}$ " DNG cylinders having the electric line source placed at $b=2 \lambda_{0}$ (see Figure 10 for the corresponding near-field results). In the case of the $2 \lambda_{0}$ distance from the cylinder, the maximum directivity for the DPS cylinders was somewhat lower than in the case of the $1 \lambda_{0}$ distance.

Moreover, the shadow region for DNG cylinders was considerably narrowed, as expected, relative to the $1 \lambda_{0}$-distance cases considered above.

For cylinders of other material parameters for which the results are not included in this manuscript, the observations regarding the directivity resembled those presented above.

\subsubsection{Radiation Resistance}

In Figure 19, the results for $R_{\text {rad }}^{\text {norm }}$ are shown for " $1 \varepsilon_{0} 1 \mu_{0}$," " $2 \varepsilon_{0} l \mu_{0}$," and " $1 \varepsilon_{0} 2 \mu_{0}$ " DPS and DNG cylinders as a function of the cylinder's radius, $a$, where $a \in[0.01,10] \lambda_{0}$, for the electric line source located at $b=1 \lambda_{0}$. In Figure 20, the results for $R_{\text {rad }}^{\text {norm }}$ are shown for " $1 \varepsilon_{0} 1 \mu_{0}, "$ " $2 \varepsilon_{0} 1 \mu_{0}$," and " $1 \varepsilon_{0} 2 \mu_{0}$ " DPS and DNG cylinders of radius $a=2 \lambda_{0}$ as a function of the distance to the electric line source, $b$, where $b \in[0.25,0.75] \lambda_{0}$.

As could be observed, there was of course no variation for the " $1 \varepsilon_{0} 1 \mu_{0}$ " DPS cylinders (see Figure 19a), while the case of the " $1 \varepsilon_{0} 1 \mu_{0}$ " DNG cylinders was seen to exhibit some variation of $R_{\text {rad }}^{\text {norm }}$ as $a$ changed. For the cylinders having the two other sets of material parameters (see Figures $19 \mathrm{~b}$ and $19 \mathrm{c}$ ), $R_{\text {rad }}^{\text {norm }}$ was seen to slightly vary with $a$. However, the variation was somewhat more pronounced for DNG than for DPS cylinders.

The variation in $R_{\text {rad }}^{\text {norm }}$ was also observed in the case of varying distance to the electric line source. While the results for the " $1 \varepsilon_{0} 1 \mu_{0}$ " DPS cylinder were as expected, the " $1 \varepsilon_{0} 1 \mu_{0}$ " DNG cylinder exhibited a rather pronounced variation of $R_{\text {rad }}^{\text {norm }}$ with $b$. In addition, the " $2 \varepsilon_{0} 1 \mu_{0}$ " and " $1 \varepsilon_{0} 2 \mu_{0}$ " DPS cylinders did not show a variation as large as did the corresponding DNG cylinders. However, the variation was more pronounced for the " $1 \varepsilon_{0} 2 \mu_{0}$ " DNG cylinder than for the " $2 \varepsilon_{0} 1 \mu_{0}$ " DNG cylinder, and in both cases the variation was larger for DNG than for DPS cylinders.

For cylinders of other material parameters of which the results are not included in this manuscript, the observations regarding the radiation resistance resembled those presented above.

\section{Conclusions}

In the present work, some properties of a general antenna configuration consisting of an infinitely long circular cylinder of lossless DNG material, illuminated by a close-by electric line source, have been investigated. The analytical solution was first derived for the case of DPS cylinders, and the steps necessary to obtain the solution for DNG cylinders were then outlined. In this regard, the sign of some important parameters for DNG materials was briefly discussed. Subsequently, numerical calculations were performed to investigate the influence of different geometrical and electromagnetic parameters on the near field, inside as well as outside the cylinder, and on the far field in terms of the directivity and radiation resistance.

It was observed that relative to the results of DPS cylinders, the effect of the DNG cylinder was most notable in the case of " $1 \varepsilon_{0} 1 \mu_{0}$ " cylinders, for which the near-field results revealed a formation of a field pattern with several lobes and a shadow region. Moreover, a high-field-value formation was observed at a point inside the DNG cylinders, suggesting that some focusing of the field was taking place. It was furthermore noted that the point of the high-field formation did not represent an exact image of the electric line source, due to the curved surface of the cylinder and its finite aperture. Similar features were observed for " $2 \varepsilon_{0} 1 \mu_{0}$ " and " $1 \varepsilon_{0} 2 \mu_{0}$ " DNG cylinders, but the high field value at a point inside the cylinders was found to be clearer. The presence of a localized high field value for the DNG cylinders was shown to be supported by the GO ray-tracing results. No distinct main lobe could be observed in the pattern for the " $2 \varepsilon_{0} 1 \mu_{0}$ " and " $1 \varepsilon_{0} 2 \mu_{0}$ "DNG cylinders, while the corresponding DPS cylinders were found to be directive, with a clear main lobe. However, for specific small distances to the electric line source, a constructive interference was observed in the pattern for " $1 \varepsilon_{0} 1 \mu_{0}$ " DNG cylinders, and this led to a sort of a main lobe, while destructive interference was observed for some other distances. The same phenomena, but far less pronounced, was observed for the " $2 \varepsilon_{0} 1 \mu_{0}$ " and " $1 \varepsilon_{0} 2 \mu_{0}$ " DNG cylinders. It was furthermore shown that the near-field resuits were well in agreement with the directivity, which was rather ripple-like and without a distinct main lobe for the DNG cylinders. In contrast to this, the directivity of the " $2 \varepsilon_{0} 1 \mu_{0}$ " and " $1 \varepsilon_{0} 2 \mu_{0}$ " DPS cylinders was observed to posses a clear main lobe, while, of course, being uniform for the " $1 \varepsilon_{0} 1 \mu_{0}$ " DPS cylinders. The radiation resistance varied more for DNG cylinders than for DPS cylinders as a function of their radius (" $1 \varepsilon_{0} 1 \mu_{0}$ " DPS cylinders exhibited, of course, no variation). This was also the case when the variation with the distance to the electric line source was investigated.

In conclusion, the near- as well as far-field properties of DNG cylinders, illuminated by an electric line source, were found to be vastly different from those of the corresponding DPS cylinders. While a DPS cylinder can function as a lens and provide a directive pattern with a distinct main lobe, this is not the case for the DNG cylinder configurations investigated herein. Other antenna-like configurations have been investigated in the literature [12-19], and many more configurations are obviously possible. An extension of the present work will involve DNG materials that are lossy and dispersive. In addition, an investigation into the near field, directivity, and radiation resistance of PEC or DPS cylinders covered with lossless and lossy DNG cylindrical shells will be conducted. 


\section{References}

1. V. G. Veselago, "The Electrodynamics of Substances with Simultaneously Negative Values of $\varepsilon$ and $\mu$," Sov. Phys. Usp., 10, January-February 1968, pp. 509-514.

2. D. R. Smith, W. J. Padilla, D. C. Vier, S. C. Nemat-Nasser, and S. Schultz, "Composite Medium with Simultaneously Negative Permeability and Permittivity," Phys. Rev. Lett., 84, May 2000, pp. 4184-4187.

3. R. A. Shelby, D. R. Smith, S. C. Nemat-Nasser, and S. Schultz, "Microwave Transmission Through a Two-Dimensional, Isotropic, Left-Handed Metamaterial," App. Phys. Lett., 78, January 2001, pp. 489-491.

4. J. B. Pendry, A. J. Holden, W. J. Stewart, and I. Youngs, "Extremely Low Frequency Plasmons in Metallic Meso Structures," Phys. Rev. Lett., 76, June 1996, pp. 4773-4776.

5. J. B. Pendry, A. J. Holden, D. J. Robbins, and W. J. Stewart, "Magnetism from Conductors and Enhanced Nonlinear Phenomena," IEEE Trans. Microwave Theory Tech., MTT-47, November 1999 , pp. 2075-2084.

6. R. A. Shelby, D. R. Smith, and S. Schultz, "Experimental Verification of a Negative Index Of Refraction," Science, 292, April 2001, pp. 77-79.

7. C. G. Parazzoli, R. B. Greegor, K. Li, B. E. C. Koltenbah, and M. Tanielian, "Experimental Verification and Simulation of Negative Index of Refraction Using Snell's Law," Phys. Rev. Lett., 90, March 2003, pp. 4011-4014.

8. R. Marques, J. Martel, F. Mesa, and F. Medina, "A New 2D Isotropic Left-Handed Metamaterial Design: Theory and Experiment," Microwave Opt. Tech. Lett., 35, December 2002, pp. 405-408.

9. G. V. Eleftheriades, A. K. Iyer, and P. C. Kremer, "Planar Negative Refractive Index Media Using Periodically L-C Loaded Transmission Lines," IEEE Trans. Microwave Theory Tech., MTT-50, December 2002, pp. 2702-2712.

10. C. Caloz, and T. Itoh, "Transmission Line Approach of Left-Handed (LH) Materials and Microstrip Implementation of an Artificial LH Transmission Line," IEEE Trans. Antennas Propagat., AP-52, May 2004, pp. $1159-1166$.

11. C. L. Holloway, E. F. Kuester, J. Baker-Jarvis, and P. Kabos, "A Double Negative (DNG) Composite Medium Composed of Magnetodielectric Spherical Particles Embedded in a Matrix,"IEEE Trans. Antennas Propagat., AP-51, October 2003, pp. 2596-2603.

12. J. B. Pendry, "Negative Refraction Makes a Perfect Lens," Phys. Rev. Let., 85, October 2000, pp. 3966-3969.

13. V. Kuzmiak and A. A. Maradudin, "Scattering Properties of a Cylinder Fabricated from a Left-Handed Material," Phys. Rev. B., 66, July 2002 , pp. 1161-1167.

14. A. Alù and N. Engheta, "Resonances in Sub-Wavelength Cylindrical Structures Made of Pairs of Double-Negative and Double-Positive or $\varepsilon$-Negative and $\mu$-Negative Coaxial Shells," Dig. of ICEAA' 03, Turin, Italy, September 8-12, 2003, pp. 435-438.
15. R. Ruppin, "Surface Polaritons and Extinction Properties of a Left-Handed Cylinder," J. Phys.: Condens. Matter, 16, August 2004, pp. 5991-5998.

16. R. Ruppin, "Intensity Distribution Inside Scatterers with Negative-Real Permittivity and Permeability," Microwave Opt. Technol. Lett., 36, pp. 150-154, February 2003.

17. R. Ruppin, "Extinction Properties of a Sphere with Negative Permittivity and Permeability," Solid State Commun., 116, August 2000, pp. 411-415.

18. R. W. Ziolkowski and A. Kipple, "Application of Double Negative Materials to Increase the power Radiated by Electrically Small Antennas," IEEE Trans. Antennas Propagat., AP-51, October 2003, pp. 2626-2640.

19. Z. Liu, Z. Lin and S. T. Chui, "Electromagnetic Scattering by Spherical Negative-Refractive-Index Particles: Low-Frequency Resonance and Localization Parameters," Phys. Rev. E., 69, January 2004, pp. 016 619/1-6.

20. M. Duncan (ed.), "Focus Issue: Negative Refraction and Metamaterials," Optic Express, On-line, 11, April 2003.

21. R. W. Ziolkowski and N. Engheta (eds.), "Special Issue on Metamaterials," IEEE Trans. Antennas Propagat., AP-51, October 2003.

22. J. R. Wait, Eleciromagnetic Radiation from Cylindrical Structures, London, Pergamon Press, Inc., 1959.

23. M. Kerker, Scattering of Light and Other Electromagnetic Radiation, New York, Academic Press, Inc., 1969.

24. C. A. Balanis, Advanced Engineering Electromagnetics, New York, John Wiley \& Sons, Inc., 1989.

25. W. C. Chew, Waves and Fields in Inhomogeneous Media, New York, IEEE Press, Inc., 1995.

26. D. R. Smith and N. Kroll, "Negative Refractive Index in Left-Handed Materials," Phys. Rev. Lett., 85, October 2000, pp. 2933-2936.

27. R. W. Ziolkowski and E. Heyman, "Wave Propagation in Media Having Negative Permittivity and Permeability," Phys. Rev, E., 64, October 2001, pp. 056 625/1-15.

28. 1. V. Lindel1, S. A. Tretyakov, K. I. Nikoskinen, and S. Iivonen, "BW Media - Media with Negative Parameters, Capable of Supporting Backward Waves," Microwave Opt. Technol. Left., 31, October 2001, pp. 129-133.

29. A. L. Pokrovsky and A. L. Efros, "Sign of Refractive Index and Group Velocity in Left-Handed Media," Solid State Commun., 124, 2002, pp. 283-287.

30. S. Arslanagić and O. Breinbjerg, "A Note on the Sign of Some Important Parameters for Left-Handed Materials," Ørsted - DTU, Electromagnetic Systems, Technical University of Denmark, Internal Report, IR 781, July 2004.

31. S. Arslanagic and O. Breinbjerg, "Clarification of the Sign of Wave Number, Intrinsic Impedance, and Refractive Index for Sim- 
ple Lossless and Lossy Double Negative Materials," Negative Refraction: Revisiting Electromagnetics from Microwaves to Optics, Laussane, Switzerland, February-March 2005, p. 78.

32. M. Abramowitz and I, A. Stegun, Handbook of Mathematical Functions, Mineola, NY, Dover Publications, Inc., 1965.

\section{Introducing the Feature Article Authors}

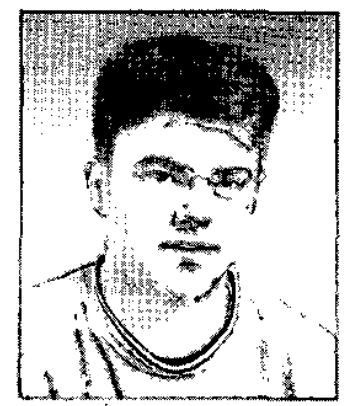

Samel Arslanagić was born in Sarajevo, Bosnia and Herzegovina, on July 28,1979 . He received the MScEE from the Technical University of Denmark (DTU) in 2004, and he is currently pursuing the $\mathrm{PhD}$ degree at the same university. In the fall of 2005, he was a Visiting Scholar at the Electromagnetics Laboratory, Tucson, Arizona, USA, where he worked on wave interaction with metamaterials.

Samel Arslanagic's main research interests include all aspects of applied electromagnetic field theory, this being particularly the case for the topics related to antennas, scattering, and metamaterials.
Fulbright Research Scholar at the University of Texas at Austin, Texas, USA, in the spring of 1995.

Dr. Breinbjerg's research is generally in applied electromagnetics - and particularly in antennas, antenna measurements, computational techniques and scattering - for applications in wireless communication and sensing technologies. At present, his antenna interests focus on application of double-negative materials and phased arrays. Contributions have been made to the theory and practice of spherical near-field antenna measurements, in particular relating to high-accuracy measurements, higher-order probes, and antenna diagnostics. Several contributions have been made to high-frequency asymptotic techniques, such as Physical Optics (PO), Physical Theory of Diffraction (PTD), and Geometrical Theory of Diffraction (GTD). In computational electromagnetics, contributions have been made to techniques such as Integral Equation/Method of Moments and Method of Auxiltary Sources (MAS): e.g., the use of MAS for impedance surfaces. He is the author or coauthor of more than 150 journal papers, conference papers, and technical reports.

Dr. Breinbjerg received a US Fulbright Research Award in 1995. He also received the 2001 AEG Elektron Foundation's Award in recognition of his research in applied electromagnetics. Furthermore, he received the 2003 DTU Student Union's Teacher of the Year Award for his course on electromagnetics. (18)

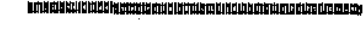

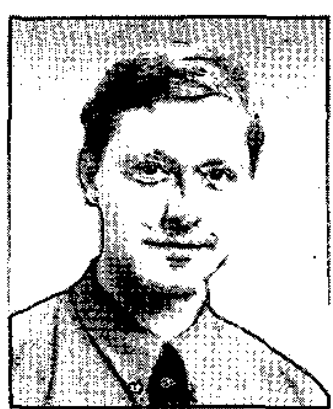

Olav Breinbjerg received the $\mathrm{MScEE}$ and $\mathrm{PhD}$ degrees from the Technical University of Denmark (DTU) in 1987 and 1992, respectively. He has been on the faculty of Orsted-DTU, Electromagnetic Systems section since 1991, where he became full Professor in January, 2006. Since 1997, he has been Head of the Antenna and Electromagnetics Group, and since 2000, also Head of the DTU-ESA Spherical Near-Field Antenna Test Facility. Since 2001, he has been Coordinator of the DTU Electrical Engineering Line, He has been a Member of the Executive Board of the EU Network of Excellence, Antenna Centre of Excellence, since 2004; a Member of the Danish Strategic Research Council's Programme Committee for Non-Ionizing Radiation since 2004; and a Member of the URSI Danish National Committee since 2004, Olav Breinbjerg was a Visiting Scientist at Rome Laboratory, Hanscom Air Force Base, Massachusetts, USA, in the fall of 1988, and a

\section{Changes of Address or Delivery Problems}

Information regarding subscription addresses is managed by IEEE headquarters. It is not maintained, nor can it be changed, by any member of the Magazine staff. If you are a member of the IEEE, your subscription is sent to the address in your IEEE member record. Your record can be updated via the Web at http://www.ieee.org/membership/coa.html. This can also be done by contacting IEEE headquarters: Member Address Records, IEEE Headquarters, 445 Hoes Lane, Piscataway NJ 08855-1331 USA; Tel: +1 (908) 981-0060 or +1 (800) 679-4333; Fax: +1 (908) 9819667; E-mail: address.change@ieee.org. If you are an institutional or other nonmember subscriber, contact IEEE Customer Service at the above address, telephone, and fax numbers; E-mail: customer. service@ieee.org.

A Web site is also available for anyone experiencing delivery problems: http:/www.ieee.org/pubdeliveryR9. If the steps suggested on the Web site do not resolve the problem, please fill out the online form.

Please do not send requests related to the above items to any member of the Magazine staff. 